\title{
On well-posedness and concentration of blow-up solutions for the intercritical inhomogeneous NLS equation
}

\author{
Mykael Cardoso, Luiz Gustavo Farah and Carlos M. Guzmán
}

\begin{abstract}
We consider the focusing inhomogeneous nonlinear Schrödinger (INLS) equation in $\mathbb{R}^{N}$

$$
i \partial_{t} u+\Delta u+|x|^{-b}|u|^{2 \sigma} u=0,
$$

where $N \geqslant 2$ and $\sigma, b>0$. We first obtain a small data global result in $H^{1}$, which, in the two spatial dimensional case, improves the third author result in [22] on the range of $b$. For $N \geqslant 3$ and $\frac{2-b}{N}<\sigma<\frac{2-b}{N-2}$, we also study the local well posedness in $\dot{H}^{s_{c}} \cap \dot{H}^{1}$, where $s_{c}=\frac{N}{2}-\frac{2-b}{2 \sigma}$. Sufficient conditions for global existence of solutions in $\dot{H}^{s_{c}} \cap \dot{H}^{1}$ are also established, using a Gagliardo-Nirenberg type estimate. Finally, we study the $L^{\sigma_{c}}$-norm concentration phenomenon, where $\sigma_{c}=\frac{2 N \sigma}{2-b}$, for finite time blow-up solutions in $\dot{H}^{s_{c}} \cap \dot{H}^{1}$ with bounded $\dot{H}^{s_{c}}$-norm. Our approach is based on the compact embedding of $\dot{H}^{s_{c}} \cap \dot{H}^{1}$ into a weighted $L^{2 \sigma+2}$ space.
\end{abstract}

\section{Introduction}

In this paper, we study the initial value problem (IVP) for the inhomogeneous nonlinear Schrödinger (INLS) equation

$$
\left\{\begin{array}{l}
i \partial_{t} u+\Delta u+|x|^{-b}|u|^{2 \sigma} u=0, \quad x \in \mathbb{R}^{N}, t>0, \\
u(x, 0)=u_{0}(x),
\end{array}\right.
$$

where $\sigma, b>0$ are real numbers.

The INLS model is a extension of the classical nonlinear Schrödinger equation (case $b=0$ ), extensively studied in recent years (see, Sulem and Sulem [34] Bourgain [2], Cazenave [5], Linares and Ponce [27], Tao [35], Fibich [16] and the references therein). As suggested by Gill [19] and Liu and Tripathi [28], it can be used as a model for the propagation of laser beams in nonlinear optics.

The well-posedness for the INLS equation $H^{1}\left(\mathbb{R}^{N}\right)$ was first studied by Genoud and Stuart [18]. Specifically, they showed that (1.1) is locally well-posed in $H^{1}\left(\mathbb{R}^{N}\right)$ if $0<\sigma<\frac{2-b}{N-2}(0<\sigma<\infty$, if $N=1,2)$ and $0<b<2$. The $H^{1}$ flow admits the conservation of mass $M[u]$ and energy $E[u]$ defined by

$$
\begin{gathered}
M[u(t)]=\int|u(t)|^{2} d x=M\left[u_{0}\right], \\
E[u(t)]=\frac{1}{2} \int|\nabla u(t)|^{2} d x-\frac{1}{2 \sigma+2} \int|x|^{-b}|u(t)|^{2 \sigma+2} d x=E\left[u_{0}\right] .
\end{gathered}
$$

An important symmetry is given by the scaling

$$
u_{\rho}(x, t)=\rho^{\frac{2-b}{2 \sigma}} u\left(\rho x, \rho^{2} t\right), \quad \rho>0 .
$$

It is easy to see that if $u(x, t)$ is a solution to the INLS equation, then $u_{\rho}(x, t)$ is also a solution.

The critical Sobolev index $s_{c}$ related to (1.1) is such that the homogeneous Sobolev space $H^{s_{c}}\left(\mathbb{R}^{N}\right)$ leaves the scaling symmetry invariant, explicitly

$$
s_{c}=\frac{N}{2}-\frac{2-b}{2 \sigma} .
$$

We say that the problem is mass-critical (or $L^{2}$-critical) if $s_{c}=0$, energy-critical (or $\dot{H}^{1}$-critical) if $s_{c}=1$ and intercritical if $0<s_{c}<1$. In terms of $\sigma$ and $b$ we can reformulate these condition as 
Mass-critical: $\sigma=\frac{2-b}{N}$;

Energy-critical ${ }^{1}: \sigma=\frac{2-b}{N-2}$;

Intercritical: $\frac{2-b}{N}<\sigma<\frac{2-b}{N-2}\left(\frac{2-b}{N}<\sigma<\infty\right.$, if $\left.N=1,2\right)$.

Several other authors studied the well-posedness of the IVP (1.1). Genoud [17] studied the masscritical case and showed the global well-posedness in $H^{1}\left(\mathbb{R}^{N}\right), N \geqslant 1$, provided that the mass of the initial data staisfies an appropriate smallness condition. This result was extended to the intercritical case by the second author in [12]. Applying a different method, based on the Strichartz estimates satisfied by the linear evolution, the third author in [22] established the local well-posedness in $H^{1}\left(\mathbb{R}^{N}\right)$, for $N \geqslant 2,0<\sigma<\frac{2-b}{N-2}(0<\sigma<\infty$, if $N=2)$ and $0<b<\frac{N}{3}$, if $N=2,3$ or $0<b<2$, if $N \geqslant 4$. In addition, in the intercritical case, he also showed a small data global theory in $H^{1}\left(\mathbb{R}^{N}\right)$ for $N \geqslant 2$ with the same assumptions on the parameter $b$. For the local theory in $H^{1}\left(\mathbb{R}^{N}\right)$, the range of $b$ was extended by Dinh $[9]^{2}$ in dimension $N=2$ to $0<b<1$ and by Cho and Lee $[6]^{3}$ in dimension $N=3$ to $0<b<\frac{3}{2}$. In all these results the range of $b$ is more restricted than the one in Genoud and Stuart [18], where the authors considered $0<b<2$. However, the works [22], [9] and [6] obtained the extra information that the solutions belong to the spaces $\in L^{q}\left([-T, T] ; L^{r}\right)$ for any $L^{2}$-admissible pair $(q, p)$ satisfying

$$
\frac{2}{q}=\frac{N}{2}-\frac{N}{p}
$$

where

$$
\left\{\begin{array}{l}
2 \leqslant p \leqslant \frac{2 N}{N-2} \quad \text { if } \quad N \geqslant 3 \\
2 \leqslant p<\infty \quad \text { if } \quad N=2 \\
2 \leqslant p \leqslant \infty \quad \text { if } \quad N=1
\end{array}\right.
$$

More recently, Campos [3] also proved a small data global theory in $H^{1}\left(\mathbb{R}^{N}\right)$ in the intercritical regime improving the range of $b$ to $0<b<3 / 2$ in dimension $N=3$. Inspired by this last result, our first goal of this paper, is to improve the small data global result of [22] in the intercritical $2 \mathrm{D}$ to the whole range of $b$ where local well-posedness was obtained by [9], that is $0<b<1$. More precisely.

Theorem 1.1. Assume $N=2$ and $0<b<1$. Suppose $\frac{2-b}{2}<\sigma<\infty$ and $u_{0} \in H^{1}\left(\mathbb{R}^{N}\right)$ satisfies $\left\|u_{0}\right\|_{H^{1}} \leqslant \eta$, for some $\eta>0$. Then there exists $\delta=\delta(\eta)>0$ such that if $\left\|e^{i t \Delta} u_{0}\right\|_{S\left(\dot{H}^{s_{c}}\right)}<\delta$, then there exists a unique global solution $u$ of (1.1) such that ${ }^{4}$

$$
\|u\|_{S\left(\dot{H}^{s_{c}}\right)} \leqslant 2\left\|e^{i t \Delta} u_{0}\right\|_{S\left(\dot{H}^{s_{c}}\right)} \quad \text { and } \quad\|u\|_{S\left(L^{2}\right)}+\|\nabla u\|_{S\left(L^{2}\right)} \leqslant 2 c\left\|u_{0}\right\|_{H^{1}},
$$

for some universal constant $c>0$.

Other issues, such as, scattering, minimal mass blow-up solutions and concentration were also investigated for the INLS equation. The second and third authors in [13]-[14] and Campos [3] proved, for different ranges on the parameter $N, \sigma$ and $b$, that radial solutions of the IVP (1.1) scatter in $H^{1}\left(\mathbb{R}^{N}\right)$ in the intercritical case. The radial assumption was removed by Miao, Murphy and Zheng [31] in the 3D cubic setting. Combet and Genoud [7] obtained the classification of minimal mass finite time blow-up solutions for mass-critical INLS equation. Note that Genoud [17] proved the existence of minimal mass finite time blow-up solutions based on the pseudo-conformal transformation applied to a standing wave solution. Finally, Campos and the first author [4] studied, also in the mass-critical case, the $L^{2}$-norm concentration of finite time blow-up solutions.

Another main purpose of this work is to study some dynamical properties of the blow-up solutions to (1.1) with initial data in $\dot{H}^{s_{c}}\left(\mathbb{R}^{N}\right) \cap \dot{H}^{1}\left(\mathbb{R}^{N}\right), 0<s_{c}<1$. To this end, we first need a local theory in this space, since, in view of the lack of mass conservation, this is not a trivial consequence of the local theory in $H^{1}\left(\mathbb{R}^{N}\right)$. We prove the following result.

\footnotetext{
${ }^{1}$ Note that this case is only possible if $N \geqslant 3$.

${ }^{2}$ Dinh also improved considered the case $N=3$ and $\frac{1}{2}<b<\frac{3}{2}$, however with the extra assumption $\sigma<\frac{3-2 b}{2 b-1}$.

${ }^{3}$ It is worth mentioning that Cho-Lee studied the INLS equation with a potencial $i \partial u_{t}+\Delta u+V u+|x|^{-b}|u|^{2 \sigma} u=0$.

${ }^{4}$ Here, as usual, $e^{i t \Delta}$ denotes the unitary group associated with the linear Schrödinger equation $i \partial_{t} u+\Delta u=0$.
} 
Theorem 1.2. Let $N \geqslant 3,0<b<\min \left\{\frac{N}{2}, 2\right\}$ and $\frac{2-b}{N}<\sigma<\frac{2-b}{N-2}$. If $u_{0} \in \dot{H}^{s_{c}}\left(\mathbb{R}^{N}\right) \cap \dot{H}^{1}\left(\mathbb{R}^{N}\right)$, then there exist $T>0$ and a unique solution u to (1.1) satisfying

$$
u \in C\left([-T, T] ; \dot{H}^{s_{c}} \cap \dot{H}^{1}\right) \bigcap L^{q}\left([-T, T] ; \dot{H}^{s_{c}, p} \cap \dot{H}^{1, p}\right) \bigcap L^{a}\left([-T, T] ; L^{r}\right),
$$

for any $(q, p) L^{2}$-admissible and $(a, r) \dot{H}^{s_{c}}$-admissible ${ }^{5}$.

To prove Theorems 1.1-1.2, we use the contraction mapping argument based on the Strichartz estimates related to the linear problem. Here and in what follows, by a solution of the IVP (1.1) with $u_{0} \in X\left(X=H^{1}\left(\mathbb{R}^{N}\right)\right.$ or $\left.X=\dot{H}^{s_{c}}\left(\mathbb{R}^{N}\right) \cap \dot{H}^{1}\left(\mathbb{R}^{N}\right)\right)$, we mean a function $u \in C(I ; X)$ on some interval $I \ni 0$ that satisfies the Duhamel formula given by

$$
u(t)=e^{i t \Delta} u_{0}+i \int_{0}^{t} e^{i\left(t-t^{\prime}\right) \Delta}|x|^{-b}|u|^{2 \sigma} u\left(t^{\prime}\right) d t^{\prime}, \quad \text { for } \quad t \in I .
$$

It is worth noticing that the local theory stated in Theorem 1.2 also holds for the defocusing inhomogeneous nonlinear Schrödinger (INLS) equation $i \partial_{t} u+\Delta u-|x|^{-b}|u|^{2 \sigma} u=0$. Moreover, it is still an open problem to obtain the same result for $N=1,2$ in both focusing and defocusing cases.

After we established the local theory in $\dot{H}^{s_{c}}\left(\mathbb{R}^{N}\right) \cap \dot{H}^{1}\left(\mathbb{R}^{N}\right)$ for the intercritical INLS equation, we study the asymptotic behavior of the solutions. We recall that Campos and the first author in [4] established, using a Sobolev embedding (see Stein-Weiss [33, Theorem B*]), the following inequality

$$
\int|x|^{-b}|f|^{2 \sigma+2} d x \leqslant c\|\nabla f\|_{L^{2}}^{2}\|f\|_{L^{\sigma_{c}}}^{2 \sigma}
$$

for some $c>0$ and all functions $f \in \dot{H}^{1}\left(\mathbb{R}^{N}\right) \cap L^{\sigma_{c}}\left(\mathbb{R}^{N}\right)$. First, we obtain the best constant for the above inequality. More precisely, we have the following sharp Gagliardo-Nirenberg type estimate.

Theorem 1.3. Let $N \geqslant 1,0<b<2, \frac{2-b}{N}<\sigma<\frac{2-b}{N-2}\left(\frac{2-b}{N}<\sigma<\infty\right.$, if $\left.N=1,2\right)$ and $\sigma_{c}=\frac{2 N \sigma}{2-b}$, then the following Gagliardo-Nirenberg inequality holds for all $f \in \dot{H}^{1}\left(\mathbb{R}^{N}\right) \cap L^{\sigma_{c}}\left(\mathbb{R}^{N}\right)$

$$
\int_{\mathbb{R}^{N}}|x|^{-b}|f(x)|^{2 \sigma+2} d x \leqslant \frac{\sigma+1}{\|V\|_{L^{\sigma_{c}}}^{2 \sigma}}\|\nabla f\|_{L^{2}}^{2}\|f\|_{L^{\sigma_{c}}}^{2 \sigma},
$$

where $V$ is a solution to the elliptic equation,

$$
\Delta V+|x|^{-b}|V|^{2 \sigma} V-|V|^{\sigma_{c}-2} V=0
$$

with minimal $L^{\sigma_{c}-n o r m}$.

Although uniqueness of solutions for the elliptic equation (1.5) is not known, this will not be an issue to our purpose since the sharp constant depends only on the $L^{\sigma_{c}}$-norm of the solution. The proof of Theorem 1.3 relies mainly in a compact embedding result (see Proposition 4.2 below) generalizing the one obtained by Genoud and Stuart [18]. Moreover, since $\dot{H}^{s_{c}}\left(\mathbb{R}^{N}\right) \subset L^{\sigma_{c}}\left(\mathbb{R}^{N}\right)$, Theorem 1.3 allows us to establish sufficient conditions for global existence in $\dot{H}^{s_{c}}\left(\mathbb{R}^{N}\right) \cap \dot{H}^{1}\left(\mathbb{R}^{N}\right)$.

Theorem 1.4. Let $N \geqslant 3,0<b<\min \left\{\frac{N}{2}, 2\right\}, \frac{2-b}{N}<\sigma<\frac{2-b}{N-2}, s_{c}=\frac{N}{2}-\frac{2-b}{2 \sigma}$ and $\sigma_{c}=\frac{2 N \sigma}{2-b}$. For $u_{0} \in \dot{H}^{s_{c}}\left(\mathbb{R}^{N}\right) \cap \dot{H}^{1}\left(\mathbb{R}^{N}\right)$, let $u(t)$ be the corresponding solution to (1.1) given by Theorem 1.2 and $T^{*}>0$ the maximal time of existence. Suppose that $\sup _{t \in\left[0, T^{*}\right)}\|u(t)\|_{\dot{H}^{s_{c}}}<\|V\|_{L^{\sigma_{c}}}$, where $V$ is a solution of the elliptic equation (1.5) with minimal $L^{\sigma_{c}}$-norm. Then $u(t)$ exists globally in the time.

Finally, we treat the phenomenon of $L^{\sigma_{c}}$-norm concentration in the intercritical regime for finite time blow-up solutions in $\dot{H}^{s_{c}}\left(\mathbb{R}^{N}\right) \cap \dot{H}^{1}\left(\mathbb{R}^{N}\right)$. We first recall that in $H^{1}\left(\mathbb{R}^{N}\right)$ a simple criterion for the existence of finite time blow-up solutions to (1.1) was obtained by the second author in [12]. Indeed, considering $u_{0} \in \Sigma:=\left\{f \in H^{1} ;|x| f \in L^{2}\right\}$, then the corresponding solution to (1.1) satisfies the virial identity

$$
\frac{d^{2}}{d t} \int|x|^{2}|u(x, t)|^{2}=8\left(2 \sigma s_{c}+2\right) E\left[u_{0}\right]-8 \sigma s_{c}\|\nabla u(t)\|_{L^{2}}^{2} .
$$

\footnotetext{
${ }^{5}$ See (2.3)-(2.4) below for the definition of $\dot{H}^{s_{c}}$-admissible pair.
} 
From this identity, we immediately see that, in the intercritical case, if $E\left[u_{0}\right]<0$, then the graph of $t \mapsto \int|x|^{2}|u|^{2}$ lies below a parabola whose concavity is facing down, which becomes negative in finite time. Therefore, the solution cannot exist globally and blows up in finite time. Dinh [10] proved the same result assuming radial negative energy initial data (also, when $N=1$, radial symmetry can also be removed). Since $H^{1}\left(\mathbb{R}^{N}\right) \subset \dot{H}^{s_{c}}\left(\mathbb{R}^{N}\right) \cap \dot{H}^{1}\left(\mathbb{R}^{N}\right)$, these results also ensure the existence of finite time blow-up solutions to (1.1) for some initial data $u_{0} \in \dot{H}^{s_{c}}\left(\mathbb{R}^{N}\right) \cap \dot{H}^{1}\left(\mathbb{R}^{N}\right)$.

Here, as in Guo [21], we suppose that the finite time blow-up solution to (1.1) is of type II, that is, the maximal time of existence $T^{*}>0$ is finite and the critical norm remains bounded

$$
\sup _{t \in\left[0, T^{*}\right)}\|u(t)\|_{\dot{H}^{s_{c}}}<\infty .
$$

It must be noted that Merle and Raphäel [29] showed the existence of radially symmetric finite time blow-up solutions to the NLS equation in $\dot{H}^{s_{c}}\left(\mathbb{R}^{N}\right) \cap \dot{H}^{1}\left(\mathbb{R}^{N}\right)$ that (1.6) does not occurs. Furthermore, they provided a lower bound for blow-up rate of the critical norm for these solution. However, their proof does not apply to the non-radial case and it may be possible to have finite time blow-up solutions satisfying (1.6) in this case.

As a consequence of the proof of Theorem 1.4 (see Remark 4.3), if there exists a solution $u(t)$ that blows up in finite time $T^{*}>0$ satisfying (1.6), then we must have

$$
\sup _{t \in\left[0, T^{*}\right)}\|u(t)\|_{L^{\sigma_{c}}} \geqslant\|V\|_{L^{\sigma_{c}}}
$$

where $V$ is a solution to elliptic equation (1.5) with minimal $L^{\sigma_{c}}$ - norm. This suggests us to investigate the occurrence of the $L^{\sigma_{c}}$-norm concentration for finite time blow-up solutions satisfying (1.6). Guo [21] obtained such concentration for the NLS equation in the intercritical case (and without radial symmetry), partially generalizing the results obtained by Holmer and Roudenko [24], which deals with the radial 3D cubic NLS equation (see also Campos the first author [4] for a similar result in the INLS setting).

Using a profile decomposition technique several authors has studied the critical norm concentration of finite time blow-up solutions (see e.g Hmidi and Keraani [23], Guo [21], Pigott and the second author [15], Campos and the first author [4] and Dinh [11]) for various dispersive models. However, in this work we use a different approach based on a compact embedding result (see Proposition 4.2 below). Our main result in this direction is the following.

Theorem 1.5. Let $N \geqslant 3,0<b<\min \left\{\frac{N}{2}, 2\right\}$ and $\frac{2-b}{N}<\sigma<\frac{2-b}{N-2}$. For $u_{0} \in \dot{H}^{s_{c}}\left(\mathbb{R}^{N}\right) \cap \dot{H}^{1}\left(\mathbb{R}^{N}\right)$, let $u(t)$ be the corresponding solution to (1.1) given by Theorem 1.2 and assume that it blows up in finite time $T^{*}>0$ satisfying (1.6). If $\lambda(t)>0$ is such that

$$
\lambda(t)\|\nabla u(t)\|_{L^{2}}^{\frac{1}{1-s_{c}}} \rightarrow \infty, \quad \text { as } \quad t \rightarrow T^{*}
$$

then,

$$
\liminf _{t \rightarrow T^{*}} \int_{|x| \leqslant \lambda(t)}|u(x, t)|^{\sigma_{c}} d x \geqslant\|V\|_{L^{\sigma_{c}}}^{\sigma_{c}}
$$

where $V$ is a minimal $L^{\sigma_{c}-n o r m ~ s o l u t i o n ~ t o ~ t h e ~ e l l i p t i c ~ e q u a t i o n ~(1.5) . ~}$

Remark 1.6. Two important comments about this result have to be emphasized. First, as a consequence of Theorem 1.2 and under the assumption (1.6), it is possible to deduce the existence of $\lambda(t)>0$ such that $\lambda(t) \rightarrow 0$, as $t \rightarrow T^{*}$. Indeed, $\lambda(t)=\left(T^{*}-t\right)^{\alpha}$ with $0<\alpha<\frac{1}{2}$ satisfies the assumptions of Theorem 1.5 (see Remark 3.6 below). Second, the inequality (1.7) asserts that the concentration occurs at the origin even for non radial finite time blow-up solutions satisfying (1.6). This is due to our method of proof based on the compact embedding result stated in Proposition 4.2. Recall that for the NLS equation concentration at the origin appears in the radial case (see Merle and Tsutsumi [30] and Tsutsumi [36]), however, for the non radial case Hmidi and Keraani [23] and Guo [21] obtained that the concentration occurs at some point of space (not necessary at the origin). 
We want to point out that the last two theorems are stated under the same assumptions as in the local theory in $\dot{H}^{s_{c}}\left(\mathbb{R}^{N}\right) \cap \dot{H}^{1}\left(\mathbb{R}^{N}\right)$ from Theorem 1.2. However, if one can improve the range of the parameters in Theorem 1.2, then Theorems 1.4-1.5 will be also true (with the same proof given here) in the same range. This is due to the fact that the sharp Gagliardo-Nirenberg type inequality given by Theorem 1.3 (and also the compact embedding result by Proposition 4.2 below) holds for the intercritical case in all dimensions $N \geqslant 1$ and $0<b<2$.

The rest of the paper is organized as follows. In Section 2, we introduce some notations and preliminary estimates. In Section 3, we obtain the well-posedness results stated in Theorems 1.1-1.2. In Section 4, we prove the sharp Gagliardo-Nirenberg inequality in Theorem 1.3 and use it to deduce Theorem 1.4. Section 5 is devoted to the proof of Theorem 1.5. Finally, in Section 6 we present another concentration result for special solutions of the INLS equation.

\section{Notation and Preliminaries}

We start this section by introducing the notation used throughout the paper. We use $c$ to denote various constants that may vary line by line. Let $a$ and $b$ be positive real numbers, the notation $a \lesssim b$ means that there exists a positive constant $c$ such that $a \leqslant c b$. Given a real number $r$, we use $r^{+}$and $r^{-}$to denote $r+\varepsilon$ and $r-\varepsilon$, respectively, for some $\varepsilon>0$ sufficiently small. For a subset $A \subset \mathbb{R}^{N}$, its complement is denoted by $A^{C}=\mathbb{R}^{N} \backslash A$ and the characteristic function $\chi_{A}(x)$ denotes the function that has value 1 at points of $\mathrm{A}$ and 0 at points of $A^{C}$. Given $x, y \in \mathbb{R}^{N}, x \cdot y$ denotes the usual inner product of $x$ and $y$ in $\mathbb{R}^{N}$. For a number $p \in[1, \infty]$ we denote its Hölder dual by $p^{\prime}=\frac{p}{p-1}$, satisfying $\frac{1}{p}+\frac{1}{p^{\prime}}=1$.

We use $\|f\|_{L^{p}}$ to denote the $L^{p}\left(\mathbb{R}^{N}\right)$ norm. The Schwartz class functions is denoted by $\mathcal{S}\left(\mathbb{R}^{N}\right)$. The norm in the Sobolev spaces $H^{s, p}=H^{s, p}\left(\mathbb{R}^{N}\right)$ and $\dot{H}^{s, p}=\dot{H}^{s, p}\left(\mathbb{R}^{N}\right)$, are defined, respectively, by $\|f\|_{H^{s, p}}:=\left\|J^{s} f\right\|_{L^{p}}$ and $\|f\|_{H^{s, p}}:=\left\|D^{s} f\right\|_{L^{p}}$, where $J^{s}$ and $D^{s}$ stand for the Bessel and Riesz potentials of order $s$, given via Fourier transform by $\widehat{J^{s} f}=\left(1+|\xi|^{2}\right)^{\frac{s}{2}} \widehat{f}$ and $\widehat{D^{s} f}=|\xi|^{s} \widehat{f}$. If $p=2$ we denote $H^{s, 2}$ and $\dot{H}^{s, 2}$ simply by $H^{s}$ and $\dot{H}^{s}$, respectively.

Let $q, p>0, s \in \mathbb{R}$, and $I \subset \mathbb{R}$ an interval; the mixed norms in the spaces $L_{I}^{q} L_{x}^{p}$ and $L_{I}^{q} H_{x}^{s}$ of a function $f=f(x, t)$ are defined as

$$
\|f\|_{L_{I}^{q} L_{x}^{p}}=\left(\int_{I}\|f(\cdot, t)\|_{L_{x}^{p}}^{q} d t\right)^{\frac{1}{q}} \quad \text { and } \quad\|f\|_{L_{I}^{q} H_{x}^{s}}=\left(\int_{I}\|f(\cdot, t)\|_{H_{x}^{s}}^{q} d t\right)^{\frac{1}{q}},
$$

with the usual modifications if either $q=\infty$ or $p=\infty$. When the $x$-integration is restricted to a subset $A \subset \mathbb{R}^{N}$ then the Lebesgue norm and the mixed norm will be denoted by $\|f\|_{L^{p}(A)}$ and $\|f\|_{L_{I}^{q} L_{x}^{r}(A)}$, respectively. Moreover, if $I=\mathbb{R}$ we shall use the notations $\|f\|_{L_{t}^{q} L_{x}^{p}}$ and $\|f\|_{L_{t}^{q} H_{x}^{s}}$.

We now recall some useful inequalities.

Lemma 2.1. (Sobolev embedding) Let $s>0$ and $1 \leqslant p<\infty$.

(i) If $s \in\left(0, \frac{N}{p}\right)$ then $H^{s, p}\left(\mathbb{R}^{N}\right)$ is continuously embedded in $L^{r}\left(\mathbb{R}^{\mathbb{N}}\right)$ where $s=\frac{N}{p}-\frac{N}{r}$. Moreover,

$$
\|f\|_{L^{r}} \leqslant C(N, s)\left\|D^{s} f\right\|_{L^{p}}
$$

(ii) If $s=\frac{N}{2}$ then $H^{s}\left(\mathbb{R}^{N}\right) \subset L^{r}\left(\mathbb{R}^{\mathbb{N}}\right)$ for all $r \in[2, \infty)$. Furthermore,

$$
\|f\|_{L^{r}} \leqslant c\|f\|_{H^{s}} .
$$

Proof. We refer to Bergh and Löfström [1, Theorem 6.5.1] for a complete proof (see also Linares and Ponce [27, Theorem 3.3] and Demengel and Demengel [8, Proposition 4.18]).

In particular, we have

$$
\|f\|_{L^{p}} \leqslant C(N, s)\|f\|_{\dot{H}^{s}}, \quad \text { for all } f \in \dot{H}^{s}\left(\mathbb{R}^{N}\right),
$$

where $p=\frac{2 N}{N-2 s}$. Moreover, for $s_{c}=\frac{N}{2}-\frac{2-b}{2 \sigma}$, we have $\dot{H}^{s_{c}}\left(\mathbb{R}^{N}\right) \subset L^{\sigma_{c}}\left(\mathbb{R}^{N}\right)$, where $\sigma_{c}=\frac{2 N \sigma}{2-b}=\frac{2 N}{N-2 s_{c}}$. 
Lemma 2.2. (Gagliardo-Nirenberg's inequality) Consider $1 \leqslant p, q, r \leqslant \infty$ and let $j, m$ be two integers, $0 \leqslant j<m$. If

$$
\frac{1}{q}-\frac{j}{N}=\theta\left(\frac{1}{r}-\frac{m}{N}\right)+\frac{1-\theta}{p}
$$

for some $\theta \in\left[\frac{j}{m}, 1\right]\left(\theta<1\right.$ if $r>1$ and $\left.m-j-\frac{N}{r}=0\right)$, then there exists a constant $c=c(j, m, p, q, r)$ such that

$$
\sum_{|\alpha|=j}\left\|D^{\alpha} f\right\|_{L^{q}} \leqslant c\left(\sum_{|\beta|=m}\left\|D^{\beta} f\right\|_{L^{r}}\right)^{\theta}\|f\|_{L^{p}}^{1-\theta}
$$

for all $f \in \mathcal{S}\left(\mathbb{R}^{N}\right)$.

Proof. See Cazenave [5, Theorem 1.3.7] and Nirenberg [32] .

Next, we recall some Strichartz type estimates associated to the linear Schrödinger propagator (see also Holmer and Roudenko [25], Guevara [20] and [14]). Given $s>0$, we say that a pair $(q, p)$ is $\dot{H}^{s}$-admissible if

$$
\frac{2}{q}=\frac{N}{2}-\frac{N}{p}-s
$$

where

$$
\begin{cases}\frac{2 N}{N-2 s} \leqslant p \leqslant\left(\frac{2 N}{N-2}\right)^{-}, \quad \text { if } \quad N \geqslant 3 \\ \frac{2}{1-s} \leqslant p \leqslant\left(\left(\frac{2}{1-s}\right)^{+}\right)^{\prime}, \quad \text { if } \quad N=2 \\ \frac{2}{1-2 s} \leqslant p \leqslant \infty, \quad \text { if } \quad N=1 .\end{cases}
$$

In the same way, we say that $(q, p)$ is $\dot{H}^{-s}$-admissible if

$$
\frac{2}{q}=\frac{N}{2}-\frac{N}{p}+s
$$

where

$$
\begin{cases}\left(\frac{2 N}{N-2 s}\right)^{+} \leqslant p \leqslant\left(\frac{2 N}{N-2}\right)^{-}, & \text {if } N \geqslant 3 \\ \left(\frac{2}{1-s}\right)^{+} \leqslant p \leqslant\left(\left(\frac{2}{1+s}\right)^{+}\right)^{\prime}, & \text { if } \quad N=2 \\ \left(\frac{2}{1-2 s}\right)^{+} \leqslant p \leqslant \infty, & \text { if } \quad N=1 .\end{cases}
$$

Now for $s \in \mathbb{R}$, let $\mathcal{A}_{s}=\left\{(q, p) ;(q, p) \text { is } \dot{H}^{s}-\text { admissible }\right\}^{6}$. We define the spaces $S\left(\dot{H}^{s}\right)$ and $S^{\prime}\left(\dot{H}^{-s}\right)$ equipped with the following Strichartz norm

$$
\|u\|_{S\left(\dot{H}^{s}\right)}=\sup _{(q, p) \in \mathcal{A}_{s}}\|u\|_{L_{t}^{q} L_{x}^{p}}
$$

and the dual Strichartz norm

$$
\|u\|_{S^{\prime}\left(\dot{H}^{-s}\right)}=\inf _{(q, p) \in \mathcal{A}_{-s}}\|u\|_{L_{t}^{q^{\prime}} L_{x}^{p^{\prime}}}
$$

where $\left(q^{\prime}, p^{\prime}\right)$ is the Hölder dual to $(q, p)$. We denote $S\left(\dot{H}^{0}\right)$ by $S\left(L^{2}\right)$. To indicate a restriction to a time interval $I \subset \mathbb{R}$, we will write $S\left(\dot{H}^{s} ; I\right)$ and $S^{\prime}\left(\dot{H}^{-s} ; I\right)$.

One of the main tools we use in the proof of our local and global well-posedness theory are the well-known Strichartz estimates.

Lemma 2.3. The following statements hold.

\footnotetext{
${ }^{6}$ The restriction for $(q, p) \dot{H}^{0}$-admissible is given by $(1.2)$.
} 
(i) (Linear estimates).

$$
\begin{gathered}
\left\|e^{i t \Delta} f\right\|_{S\left(L^{2}\right)} \leqslant c\|f\|_{L^{2}}, \\
\left\|e^{i t \Delta} f\right\|_{S\left(\dot{H}^{s}\right)} \leqslant c\|f\|_{\dot{H}^{s}} .
\end{gathered}
$$

(ii) (Inhomogeneous estimates).

$$
\begin{gathered}
\left\|\int_{\mathbb{R}} e^{i\left(t-t^{\prime}\right) \Delta} g\left(\cdot, t^{\prime}\right) d t^{\prime}\right\|_{S\left(L^{2}\right)}+\left\|\int_{0}^{t} e^{i\left(t-t^{\prime}\right) \Delta} g\left(\cdot, t^{\prime}\right) d t^{\prime}\right\|_{S\left(L^{2}\right)} \leqslant c\|g\|_{S^{\prime}\left(L^{2}\right)}, \\
\left\|\int_{0}^{t} e^{i\left(t-t^{\prime}\right) \Delta} g\left(\cdot, t^{\prime}\right) d t^{\prime}\right\|_{S\left(\dot{H}^{s}\right)} \leqslant c\|g\|_{S^{\prime}\left(\dot{H}^{-s}\right)} .
\end{gathered}
$$

For a complete proof we refer the reader to Linares and Ponce [27] and Kato [26] (see also Holmer and Roudenko [25], [22] and the references therein).

\section{Well-posedness theory}

In this section we prove the well-posedness results stated in Theorems 1.1-1.2. The proofs follow from a contraction mapping argument based on the Strichartz estimates. In view of the singular factor $|x|^{-b}$ in the nonlinearity, we frequently divide our analysis in two regions. Indeed, let $B=B(0,1)=$ $\left\{x \in \mathbb{R}^{N} ;|x| \leqslant 1\right\}$ a simple computation revels that

$$
\left\||x|^{-b}\right\|_{L^{\gamma}(B)}<\infty, \quad \text { if } \frac{N}{\gamma}-b>0 \text { and }\left\||x|^{-b}\right\|_{L^{\gamma}\left(B^{C}\right)}<\infty, \quad \text { if } \frac{N}{\gamma}-b<0 .
$$

We are going to use these facts several times throughout this section.

\subsection{Global well-posedness in $H^{1}\left(\mathbb{R}^{N}\right)$}

In this subsection, we turn our attention to proof the Theorem 1.1. The heart of the proof is to establish good estimates on the nonlinearity $|x|^{-b}|u|^{2 \sigma} u$. The next lemma provides these estimates.

Lemma 3.1. Let $N=2,0<b<1$ and $\frac{2-b}{2}<\sigma<\infty$. Then there exist $c>0$ and $\theta \in(0,2 \sigma)$ sufficiently small such that

(i) $\left\|\chi_{B}|x|^{-b}|u|^{2 \sigma} v\right\|_{S^{\prime}\left(\dot{H}^{-s_{c}}\right)}+\left\|\chi_{B^{C}}|x|^{-b}|u|^{2 \sigma} v\right\|_{S^{\prime}\left(\dot{H}^{-s_{c}}\right)} \leqslant c\|u\|_{L_{t}^{\infty} H_{x}^{1}}^{\theta}\|u\|_{S\left(\dot{H}^{s_{c}}\right)}^{2 \sigma-\theta}\|v\|_{S\left(\dot{H}^{s_{c}}\right)}$,

(ii) $\left\|\chi_{B}|x|^{-b}|u|^{2 \sigma} v\right\|_{S^{\prime}\left(L^{2}\right)}+\left\|\chi_{B^{C}}|x|^{-b}|u|^{2 \sigma} v\right\|_{S^{\prime}\left(L^{2}\right)} \leqslant c\|u\|_{L_{t}^{\infty} H_{x}^{1}}^{\theta}\|u\|_{S\left(\dot{H}^{s_{c}}\right)}^{2 \sigma-\theta}\|v\|_{S\left(L^{2}\right)}$,

(iii) $\left\|\nabla\left(|x|^{-b}|u|^{2 \sigma} u\right)\right\|_{L_{t}^{q^{\prime}} L_{x}^{r^{\prime}}} \leqslant c\|u\|_{L_{t}^{\infty \infty} H_{x}^{1}}^{\theta}\|u\|_{S\left(\dot{H}^{s_{c}}\right)}^{2 \sigma-\theta}\|\nabla u\|_{S\left(L^{2}\right)}+c\|u\|_{L_{t}^{\infty} H_{x}^{1}}^{\theta+1}\|u\|_{S\left(\dot{H}^{s_{c}}\right)}^{2 \sigma-\theta}$,

where $^{7}(q, r)=\left(\frac{2}{1-\theta}, \frac{2}{\theta}\right)$.

Proof. To prove $(i)$ and $(i i)$, we first define the following numbers

$$
\widehat{q}=\frac{4 \sigma(2 \sigma+2-\theta)}{2 \sigma(2 \sigma+b)-\theta(2 \sigma-2+b)}, \quad \widehat{r}=\frac{4 \sigma(2 \sigma+2-\theta)}{(2 \sigma-\theta)(2-b)}
$$

and

$$
\widehat{a}=\frac{2 \sigma(2 \sigma+2-\theta)}{2-b}, \quad \widetilde{a}=\frac{2 \sigma(2 \sigma+2-\theta)}{2 \sigma(2 \sigma+b-\theta)-(2-b)(1-\theta)} .
$$

\footnotetext{
${ }^{7}$ Note that the pair $(q, r)$ is $L^{2}$-admissible.
} 
It is easy to see that, for $\theta$ sufficiently small, $(\widehat{q}, \widehat{r})$ is $L^{2}$-admissible, $(\widehat{a}, \widehat{r})$ is $\dot{H}^{s_{c}}$-admissible and $(\widetilde{a}, \widehat{r})$ is $\dot{H}^{-s_{c}}$ admissible. Moreover

$$
\frac{1}{\widetilde{a}^{\prime}}=\frac{2 \sigma-\theta}{\widehat{a}}+\frac{1}{\widehat{a}} \quad \text { and } \quad \frac{1}{\widehat{q}^{\prime}}=\frac{2 \sigma-\theta}{\widehat{a}}+\frac{1}{\widehat{q}} .
$$

Let us prove $(i)$. Let $A \subset \mathbb{R}^{N}$ denotes either $B$ or $B^{C}$. By definition of $S^{\prime}\left(\dot{H}^{-s}\right)$, we clearly have $\left\|\chi_{A}|x|^{-b}|u|^{2 \sigma} v\right\|_{S^{\prime}\left(\dot{H}^{-s_{c}}\right)} \leqslant\left\|\chi_{A}|x|^{-b}|u|^{2 \sigma} v\right\|_{L_{t}^{\tilde{a}^{\prime}} L_{x}^{\hat{r}^{\prime}}}$. On the other hand, from Hölder's inequality we deduce

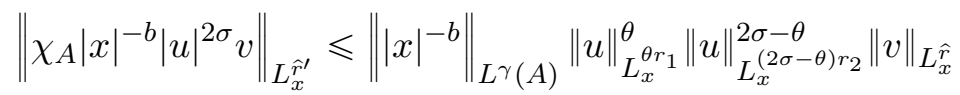

$$
\begin{aligned}
& =\left\||x|^{-b}\right\|_{L^{\gamma}(A)}\|u\|_{L_{x}^{\theta r_{1}}}^{\theta}\|u\|_{L_{x}^{\hat{r}}}^{2 \sigma-\theta}\|v\|_{L_{x}^{\hat{r}}},
\end{aligned}
$$

where

$$
\frac{1}{\widehat{r}^{\prime}}=\frac{1}{\gamma}+\frac{1}{r_{1}}+\frac{1}{r_{2}}+\frac{1}{\widehat{r}} \text { and } \widehat{r}=(2 \sigma-\theta) r_{2}
$$

Observe that (3.4) implies

$$
\frac{2}{\gamma}=2-\frac{2(2 \sigma+2-\theta)}{\widehat{r}}-\frac{2}{r_{1}}
$$

and using the value of $\widehat{r}$, it follows that

$$
\frac{2}{\gamma}-b=\frac{\theta(2-b)}{2 \sigma}-\frac{2}{r_{1}}
$$

Next we show that $\left\||x|^{-b}\right\|_{L^{\gamma}(A)}$ is finite and $H^{1} \subset L^{\theta r_{1}}$. Since $2 \sigma>2-b$ we have $\frac{4 \sigma}{2-b}>2$. Thus, if $A=B$ and choosing $\theta r_{1} \in\left(\frac{4 \sigma}{2-b}, \infty\right)$, from (3.5), we immediately get $\frac{2}{\gamma}-b>0$. Furthermore, if $A=B^{C}$ and choosing $\theta r_{1} \in\left(2, \frac{4 \sigma}{2-b}\right)$, we obtain $\frac{2}{\gamma}-b<0$. In both cases we have $\left\||x|^{-b}\right\|_{L^{\gamma}(A)}<\infty$ and, from Lemma 2.1, $H^{1} \subset L^{\theta r_{1}}$ (recall that, for $N=2$, one has $H^{1} \subset L^{p}, p \in[2, \infty)$ ). Therefore, the inequality (3.3) yields

$$
\left\|\chi_{A}|x|^{-b}|u|^{2 \sigma} v\right\|_{L_{x}^{\hat{r}^{\prime}}} \lesssim\|u\|_{H_{x}^{1}}^{\theta}\|u\|_{L_{x}^{\hat{r}}}^{2 \sigma-\theta}\|v\|_{L_{x}^{\hat{r}}}
$$

Now applying Hölder's inequality in time and recalling (3.2), we have

$$
\begin{aligned}
\left\|\chi_{A}|x|^{-b}|u|^{2 \sigma} v\right\|_{L_{t}^{\tilde{a}^{\prime}} L_{x}^{\hat{r}^{\prime}}} & \lesssim\|u\|_{L_{t}^{\infty} H_{x}^{1}}^{\theta}\|u\|_{L_{t}^{a} L_{x}^{\hat{r}}}^{2 \sigma-\theta}\|v\|_{L_{t}^{\hat{a}} L_{x}^{\hat{r}}} \\
& \lesssim\|u\|_{L_{t}^{\infty} H_{x}^{1}}^{\theta}\|u\|_{S\left(\dot{H}^{s c}\right)}^{2 \sigma-\theta}\|v\|_{S\left(\dot{H}^{s^{c}}\right)},
\end{aligned}
$$

which implies $(i)$.

Since $(\widehat{q}, \widehat{r})$ is $L^{2}$-admissible, the proof of $(i i)$ is essentially the same as $(i)$. It is worth noting that, once (3.6) is achieved, we use (3.2) to deduce

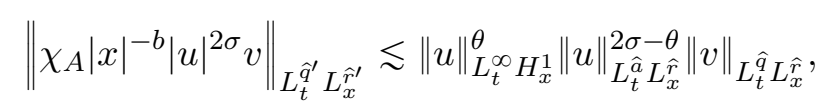

which yields $(i i)$.

Before starting the proof of $($ iii $)$, we need the following numbers

$$
\bar{a}=\frac{2(2 \sigma+1-\theta)}{1-s_{c}+\theta}, \quad \bar{r}=\frac{4 \sigma(2 \sigma+1-\theta)}{2 \sigma\left(1-b+s_{c}\right)+2-b-\theta(2-b+2 \sigma)}
$$

and

$$
\bar{q}=\frac{2(2 \sigma+1-\theta)}{1+2 \sigma s_{c}+\theta\left(1-s_{c}\right)}, \quad a^{*}=\frac{2(2 \sigma-\theta)}{1+\theta}, \quad r^{*}=\frac{4 \sigma(2 \sigma-\theta)}{2 \sigma(1-b)-\theta(2-b+2 \sigma)}
$$


Note that, for $\theta$ sufficiently small, $(\bar{q}, \bar{r})$ is $L^{2}$-admissible, $(\bar{a}, \bar{r}),\left(a^{*}, r^{*}\right)$ is $\dot{H}^{s_{c}}$-admissible and ${ }^{8}$,

$$
\frac{1}{q^{\prime}}=\frac{2 \sigma-\theta}{\bar{a}}+\frac{1}{\bar{q}} \quad \text { and } \quad(2 \sigma-\theta) q^{\prime}=a^{*} .
$$

Again, let $A \subset \mathbb{R}^{N}$ denotes either $B$ or $B^{C}$. From (3.7)) and Hölder's inequality we deduce

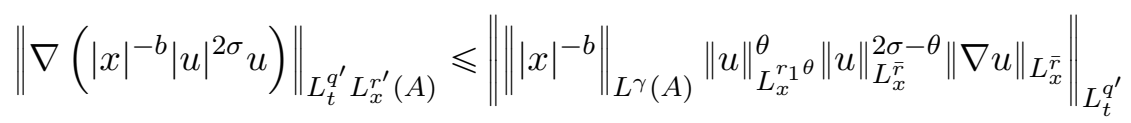

$$
\begin{aligned}
& +\|\||x|^{-b-1}\left\|_{L^{d}(A)}\right\| u\left\|_{L_{x}^{(\theta+1) p_{1}}}^{\theta+1}\right\| u\left\|_{L_{x}^{r *}}^{2 \sigma-\theta}\right\|_{L_{t}^{q^{\prime}}} \\
& \lesssim\|u\|_{L_{x}^{r_{1} \theta}}^{\theta}\|u\|_{L_{t}^{\bar{a}} L_{x}^{\bar{r}}}^{2 \sigma-\theta}\|\nabla u\|_{L_{t}^{\bar{q}} L_{x}^{\bar{x}}}+c\|u\|_{L_{x}^{(\theta+1) p_{1}}}^{\theta+1}\|u\|_{L_{t}^{a *} L_{x}^{r^{*}}}^{2 \sigma-\theta},
\end{aligned}
$$

where

$$
\frac{1}{r^{\prime}}=\frac{1}{\gamma}+\frac{1}{r_{1}}+\frac{2 \sigma-\theta}{\bar{r}}+\frac{1}{\bar{r}}=\frac{1}{d}+\frac{1}{p_{1}}+\frac{2 \sigma-\theta}{r^{*}} .
$$

Using the definition of the numbers $\bar{r}$ and $r^{*}$ one has

$$
\frac{2}{\gamma}-b=\frac{\theta(2-b)}{2 \sigma}-\frac{2}{r_{1}}, \quad \frac{2}{d}-b-1=\frac{\theta(2-b)}{2 \sigma}-\frac{2}{p_{1}},
$$

which are analogous to the relation (3.5). Finally, choosing $r_{1}$ and $p_{1}$ as in $(i)$ we have that $\left\||x|^{-b}\right\|_{L^{\gamma}(A)}$ and $\left\||x|^{-b-1}\right\|_{L^{d}(A)}$ are finite. Also, $H^{1} \subset L^{\theta r_{1}} \cup L^{(\theta+1) p_{1}}$ and we complete the proof of (iii).

It should be emphasized that the third author in [22] proved the previous lemma under the assumption $0<b<\frac{2}{3}$. Here we extend it to $0<b<1$.

Now, we have all the tools to prove Theorem 1.1.

Proof of Theorem 1.1. First note that $|x|^{-b}|u|^{2 \sigma} u=\chi_{B}|x|^{-b}|u|^{2 \sigma} u+\chi_{B^{c}}|x|^{-b}|u|^{2 \sigma} u$. So, applying Lemma 2.3, we have for $I=[-T, T]$

$$
\left\|\int_{0}^{t} e^{i\left(t-t^{\prime}\right) \Delta}|x|^{-b}|u|^{2 \sigma} u\left(t^{\prime}\right) d t^{\prime}\right\|_{S\left(L^{2} ; I\right)} \lesssim\left\|\chi_{B}|x|^{-b}|u|^{2 \sigma} u\right\|_{S^{\prime}\left(L^{2} ; I\right)}+\left\|\chi_{B^{c}}|x|^{-b}|u|^{2 \sigma} u\right\|_{S^{\prime}\left(L^{2} ; I\right)} .
$$

Similarly, we can use the same argument to estimate the norm $\|\cdot\|_{S\left(\dot{H}^{\left.s_{c} ; I\right)}\right.}$. The rest of the proof follows the same lines as in Guzmán [22, Corollary 1.12].

\subsection{Local well-posedness in $\dot{H}^{s_{c}}\left(\mathbb{R}^{N}\right) \cap \dot{H}^{1}\left(\mathbb{R}^{N}\right)$}

Recall that $s_{c}=\frac{N}{2}-\frac{2-b}{2 \sigma}$. In this subsection, we show that the IVP (1.1) is locally well-posed in $\dot{H}^{s_{c}}\left(\mathbb{R}^{N}\right) \cap \dot{H}^{1}\left(\mathbb{R}^{N}\right)$, for $N \geqslant 3$ and $\frac{2-b}{N}<\sigma<\frac{2-b}{N-2}$ (equivalently $0<s_{c}<1$ ). We start with some estimates for the gradient of the nonlinearity.

Lemma 3.2. Let $N \geqslant 3,0<b<\min \left\{\frac{N}{2}, 2\right\}$ and $\frac{2-b}{N}<\sigma<\frac{2-b}{N-2}$, then there exist $c, \theta_{1}, \theta_{2}>0$ such that the following inequality holds

$$
\left\|\chi_{B^{c}} \nabla|x|^{-b}|u|^{2 \sigma} u\right\|_{S^{\prime}\left(L^{2} ; I\right)}+\left\|\chi_{B} \nabla|x|^{-b}|u|^{2 \sigma} u\right\|_{S^{\prime}\left(L^{2} ; I\right)} \leqslant c\left(T^{\theta_{1}}+T^{\theta_{2}}\right)\|\nabla u\|_{S\left(L^{2} ; I\right)}^{2 \sigma+1},
$$

where $I=[-T, T]$.

\footnotetext{
${ }^{8}$ Since $b<1$, we have that the denominator of $r^{*}$ is positive and $\bar{r}, r^{*}>\frac{2}{1-s_{c}}$ (this is a necessary condition for $\dot{H}^{s_{c}}$-admissible pairs, see $\left.(2.4)\right)$.
} 
Proof. We start estimating the first term in the left hand side of (3.8). Define $\left(q_{0}, p_{0}\right)$ the $L^{2}$-admissible pair given $b^{9}$

$$
q_{0}=\frac{2(2 \sigma+2)}{\sigma(N-2)} \text { and } p_{0}=\frac{N(2 \sigma+2)}{N+2 \sigma},
$$

From the Hölder inequality and Sobolev inequality, it follows that

$$
\begin{aligned}
\left\|\nabla\left(|x|^{-b}|u|^{2 \sigma} u\right)\right\|_{L_{x}^{p_{0}^{\prime}\left(B^{c}\right)}} & \lesssim\left\||x|^{-b}\right\|_{L^{\gamma}\left(B^{c}\right)}\left\|\nabla\left(|u|^{2 \sigma} u\right)\right\|_{L_{x}^{\beta}}+\left\|\nabla\left(|x|^{-b}\right)\right\|_{L^{d}\left(B^{c}\right)}\|u\|_{L_{x}^{(2 \sigma+1) e}}^{2 \sigma+1} \\
& \lesssim\left\||x|^{-b}\right\|_{L^{\gamma}\left(B^{c}\right)}\|u\|_{L_{x}^{2 \sigma \alpha}}^{2 \sigma}\|\nabla u\|_{L_{x}^{p_{0}}}+\left\||x|^{-b-1}\right\|_{L^{d}\left(B^{c}\right)}\|\nabla u\|_{L_{x}^{p_{0}}}^{2 \sigma+1} \\
& \lesssim\left\||x|^{-b}\right\|_{L^{\gamma}\left(B^{c}\right)}\|\nabla u\|_{L_{x}^{p_{0}}}^{2 \sigma+1}+\left\||x|^{-b-1}\right\|_{L^{d}\left(B^{c}\right)}\|\nabla u\|_{L_{x}^{p_{0}}}^{2 \sigma+1},
\end{aligned}
$$

where the following relations are satisfied

$$
\left\{\begin{aligned}
\frac{1}{p_{0}^{\prime}} & =\frac{1}{\gamma}+\frac{1}{\beta}=\frac{1}{d}+\frac{1}{e} \\
\frac{1}{\beta} & =\frac{1}{\alpha}+\frac{1}{p_{0}} \\
1 & =\frac{N}{p_{0}}-\frac{N}{2 \sigma \alpha} ; \quad p_{0}<N \\
1 & =\frac{N}{p_{0}}-\frac{N}{(2 \sigma+1) e}
\end{aligned}\right.
$$

which are equivalent to

$$
\left\{\begin{array}{l}
\frac{N}{\gamma}=N-\frac{2 N}{p_{0}}-\frac{2 \sigma N}{p_{0}}+2 \sigma \\
\frac{N}{d}=N-\frac{2 N}{p_{0}}-\frac{2 \sigma N}{p_{0}}+2 \sigma+1
\end{array}\right.
$$

Note that, in view of (3.9) we have $\frac{N}{\gamma}-b=-b<0$ and $\frac{N}{d}-b-1=-b<0$. So, $\left\||x|^{-b}\right\|_{L^{\gamma}\left(B^{c}\right)}$ and $\left\||x|^{-b-1}\right\|_{L^{d}\left(B^{c}\right)}$ are bounded quantities (see (3.1)) and therefore

$$
\left\|\nabla\left(|x|^{-b}|u|^{2 \sigma} u\right)\right\|_{L_{x}^{p_{0}^{\prime}\left(B^{c}\right)}} \lesssim\|\nabla u\|_{L_{x}^{p_{0}}}^{2 \sigma+1}
$$

On the other hand, applying the Hölder inequality in the time variable we deduce

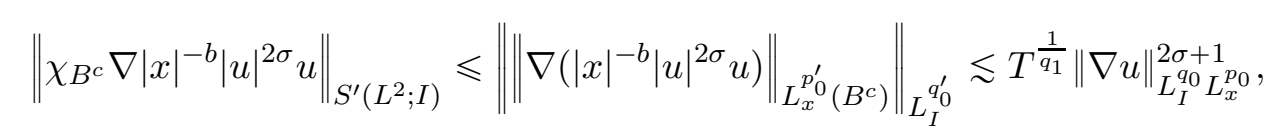

where $\frac{1}{q_{0}^{\prime}}=\frac{1}{q_{1}}+\frac{2 \sigma+1}{q_{0}}$. From (3.9), it is clear that $\frac{1}{q_{1}}=\frac{4-2 \sigma(N-2)}{4}>0$, where the positivity follows from $\sigma<\frac{2-b}{N-2}$. Setting $\theta_{1}=\frac{1}{q_{1}}$, we conclude the estimate of the first term in the left hand side of (3.8).

Next, we turn out attention to the second term in the left hand side of (3.8). First note that $\left\|\chi_{B} \nabla|x|^{-b}|u|^{2 \sigma} u\right\|_{S^{\prime}\left(L^{2} ; I\right)} \leqslant\|\| \nabla\left(|x|^{-b}|u|^{2 \sigma} u\right)\left\|_{L_{x}^{p^{\prime}(B)}}\right\|_{L_{I}^{q^{\prime}}}$. From the same arguments as in the inequality (3.10), we deduce

$$
\left\|\nabla\left(|x|^{-b}|u|^{2 \sigma} u\right)\right\|_{L_{x}^{p^{\prime}(B)}} \leqslant\left\||x|^{-b}\right\|_{L^{\gamma}(B)}\|\nabla u\|_{L_{x}^{p}}^{2 \sigma+1}+\left\||x|^{-b-1}\right\|_{L^{d}(B)}\|\nabla u\|_{L_{x}^{p}}^{2 \sigma+1},
$$

assuming (3.11) is satisfied replacing $p_{0}$ by $p$ (to be determined later), that is

$$
\left\{\begin{array}{l}
\frac{N}{\gamma}=N-\frac{2 N}{p}-\frac{2 \sigma N}{p}+2 \sigma \\
\frac{N}{d}=N-\frac{2 N}{p}-\frac{2 \sigma N}{p}+2 \sigma+1
\end{array}\right.
$$

In order to have that $\left\||x|^{-b}\right\|_{L^{\gamma}(B)}$ and $\left\||x|^{-b-1}\right\|_{L^{d}(B)}$ are bounded quantities, we need $\frac{N}{\gamma}>b$ and $\frac{N}{d}>b+1$, respectively, by (3.1). So, we want to show that $N-\frac{2 N}{p}-\frac{2 \sigma N}{p}+2 \sigma>b$. This is equivalent to $2 \sigma<\frac{(N-b) p-2 N}{N-p}$ (assuming $p<N$ ), then we choose $p$ such that

$$
\frac{(N-b) p-2 N}{N-p}=\frac{4-2 b}{N-2}
$$

\footnotetext{
${ }^{9}$ It is not difficult to check that the pair $\left(q_{0}, p_{0}\right)$ is $L^{2}$-admissible.
} 
In other words, we choose $p$ and $q$ given by ${ }^{10}$

$$
p=\frac{2 N(N-b)}{N(N-2)+4-b N} \text { and } q=\frac{2(N-b)}{N-2},
$$

where we have used that the pair $(q, p)$ is $L^{2}$-admissible to compute the value of $q$. Note that $p<N$ if, and only if, $b<N-2$. Hence, the Hölder inequality in the time variable leads to

$$
\left\|\chi_{B} \nabla|x|^{-b}|u|^{2 \sigma} u\right\|_{S^{\prime}\left(L^{2} ; I\right)} \lesssim T^{\frac{1}{q_{1}}}\|\nabla u\|_{L_{I}^{q} L_{x}^{p}}^{2 \sigma+1}
$$

where $\frac{1}{q^{\prime}}=\frac{1}{q_{1}}+\frac{2 \sigma+1}{q}$. Since $\sigma<\frac{2-b}{N-2}$, it is clear that $\frac{1}{q_{1}}=1-\frac{2 \sigma+2}{q}=\frac{4-2 b-2 \sigma(N-2)}{2(N-b)}>0$. Therefore,

$$
\left\|\chi_{B} \nabla|x|^{-b}|u|^{2 \sigma} u\right\|_{S^{\prime}\left(L^{2} ; I\right)} \lesssim T^{\theta_{2}}\|\nabla u\|_{S\left(L^{2} ; I\right)}^{2 \sigma+1},
$$

for $\theta_{2}=\frac{1}{q_{1}}>0$.

Note that the restriction $0<b<N-2$ implies that the inequality (3.13) only holds for $0<b<1$ when $N=3$. Next, we show that in dimension $N=3$ it is also possible to consider $1 \leqslant b<\frac{3}{2}$. To this end, for a pair $(q, p) L^{2}$-admissible to be chosen later, we have

$$
\begin{aligned}
& \left\|\chi_{B} \nabla|x|^{-b}|u|^{2 \sigma} u\right\|_{S^{\prime}\left(L^{2} ; I\right)} \leqslant\left\|\chi_{B} \nabla|x|^{-b}|u|^{2 \sigma} u\right\|_{L_{I}^{q^{\prime}} L_{x}^{p^{\prime}}} \\
& \leqslant\|\||x|^{-b}\left\|_{L^{\gamma}(B)}\right\| u\left\|_{L_{x}^{2 \sigma r_{1}}}^{2 \sigma}\right\| \nabla u\left\|_{L_{x}^{\bar{p}}}\right\|_{L_{I}^{q^{\prime}}}+\|\||x|^{-b-1}\left\|_{L^{d}(B)}\right\| u\left\|_{L_{x}^{(2 \sigma+1) e}}^{2 \sigma+1}\right\|_{L_{I}^{q^{\prime}}} \\
& \lesssim T^{\frac{1}{q_{1}}}\left\||x|^{-b}\right\|_{L^{\gamma}(B)}\|\nabla u\|_{L_{I}^{\bar{q}} L_{x}^{\bar{p}}}^{2 \sigma+1}+T^{\frac{1}{q_{1}}}\left\||x|^{-b-1}\right\|_{L^{d}(B)}\|\nabla u\|_{L_{I}^{\bar{q}} L_{x}^{\bar{p}}}^{2 \sigma+1}
\end{aligned}
$$

if the following conditions are satisfied

$$
\left\{\begin{array}{l}
\frac{1}{p^{\prime}}=\frac{1}{\gamma}+\frac{1}{r_{1}}+\frac{1}{\bar{p}}=\frac{1}{d}+\frac{1}{e} \\
1=\frac{3}{\bar{p}}-\frac{3}{2 \sigma r_{1}}=\frac{3}{\bar{p}}-\frac{3}{(2 \sigma+1) e}, \quad \bar{p}<3 \\
\frac{1}{q^{\prime}}=\frac{1}{q_{1}}+\frac{2 \sigma+1}{\bar{q}} .
\end{array}\right.
$$

Consider the $L^{2}$-admissible pair $(\bar{q}, \bar{p})$ given by

$$
\bar{q}=\frac{1-2 \varepsilon}{2} \text { and } \bar{p}=\frac{3}{1+\varepsilon},
$$

for $\varepsilon>0$ small enough. In this case, we have $\bar{p}<3$ and system (3.14) can be rewritten as

$$
\left\{\begin{array}{l}
\frac{3}{\gamma}-b=2-b+2 \sigma-\frac{3}{p}-\varepsilon(2 \sigma+1) \\
\frac{3}{d}-b-1=2-b+2 \sigma-\frac{3}{p}-\varepsilon(2 \sigma+1) .
\end{array}\right.
$$

Now, we need to choose $p$ such that $2-b+2 \sigma-\frac{3}{p}-\varepsilon(2 \sigma+1)>0$, and thus, by (3.1), we deduce that $\left\||x|^{-b}\right\|_{L^{\gamma(B)}}$ and $\left\||x|^{-b-1}\right\|_{L^{d(B)}}$ are bounded quantities. To this end, define the $L^{2}$-admissible $(q, p)$ given by ${ }^{11}$

$$
q=\frac{4}{2 b-1+4 \varepsilon(2 \sigma+1)} \quad \text { and } \quad p=\frac{3}{2-b-2 \varepsilon(2 \sigma+1)} .
$$

It also follows that, $\frac{1}{q_{1}}=1-\frac{1}{q}-\frac{2 \sigma+1}{\bar{q}}=1-\frac{2 b-1+4 \varepsilon(2 \sigma+1)}{4}-\frac{(2 \sigma+1)(1-2 \varepsilon)}{2}=\frac{4-2 b-2 \sigma-2 \varepsilon(2 \sigma+1)}{4}>0$ for $\varepsilon>0$ sufficient small. Therefore,

$$
\left\|\chi_{B} \nabla|x|^{-b}|u|^{2 \sigma} u\right\|_{S^{\prime}\left(L^{2} ; I\right)} \lesssim T^{\theta_{2}}\|\nabla u\|_{S\left(L^{2}\right)},
$$

for $\theta_{2}=\frac{1}{q_{1}}>0$.

Finally, collecting the last inequality, (3.12) and (3.13) we conclude the proof.

\footnotetext{
${ }^{10}$ It is easy to see that $p>2$ if, and only if, $N>2$ and $p<\frac{2 N}{N-2}$ if, and only if, $b<2$. Therefore the pair $(q, p)$ is $L^{2}$-admissible.

${ }^{11}$ Since $1 \leqslant b<\frac{3}{2}$ it is clear that $2<p<6$ and $q>0$ for $\varepsilon>0$ small enough.
} 
Lemma 3.3. Let $N \geqslant 3,0<b<\min \left\{\frac{N}{2}, 2\right\}$ and $\frac{2-b}{N}<\sigma<\frac{2-b}{N-2}$, then there exist $c, \theta_{1}, \theta_{2}>0$ such that the following inequalities hold

$$
\left\|D^{s_{c}}|x|^{-b}|u|^{2 \sigma} u\right\|_{S^{\prime}\left(L^{2} ; I\right)} \leqslant\left\|D^{s_{c}}|x|^{-b}|u|^{2 \sigma} u\right\|_{L_{I}^{2} L_{x}^{\frac{2 N}{N+2}}} \leqslant c\left(T^{\theta_{1}}+T^{\theta_{2}}\right)\|\nabla u\|_{S\left(L^{2} ; I\right)}\left\|D^{s_{c}} u\right\|_{S\left(L^{2} ; I\right)}^{2 \sigma},
$$

where $I=[-T, T]$.

Proof. The first inequality comes from the fact that the pair $\left(2, \frac{2 N}{N-2}\right)$ is $L^{2}$-admissible. Now, from Sobolev embedding (see Lemma $2.1(i)$ ) we obtain

$$
\left\|D^{s_{c}}|x|^{-b}|u|^{2 \sigma} u\right\|_{L_{x}^{\frac{2 N}{N+2}}} \lesssim\left\|\nabla|x|^{-b}|u|^{2 \sigma} u\right\|_{L_{x}^{p^{*}}},
$$

where $p^{*}=\frac{2 N \sigma}{4 \sigma+2-b}$.

Let $A \subset \mathbb{R}^{N}$ denotes either $B$ or $B^{C}$. Applying Hölder's inequality first in space and then in time, we get

$$
\begin{aligned}
\left\|\nabla|x|^{-b}|u|^{2 \sigma} u\right\|_{L_{I}^{2} L_{x}^{p^{*}}(A)} & \lesssim\|\||x|^{-b}\left\|_{L^{\gamma}(A)}\right\| u\left\|_{L_{x}^{2 \sigma \beta}}^{2 \sigma}\right\| \nabla u\left\|_{L_{x}^{p}}+\right\||x|^{-b-1}\left\|_{L^{d}(A)}\right\| u\left\|_{L_{x}^{2 \sigma}}^{2 \sigma}\right\| u\left\|_{L_{x}^{f}}\right\|_{L_{I}^{2}} \\
& \lesssim\|\||x|^{-b}\left\|_{L^{\gamma}(A)}\right\| D^{s_{c}} u\left\|_{L_{x}^{p}}^{2 \sigma}\right\| \nabla u\left\|_{L_{x}^{p}}+\right\||x|^{-b-1}\left\|_{L^{d}(A)}\right\| D^{s_{c}} u\left\|_{L_{x}^{p}}^{2 \sigma}\right\| \nabla u\left\|_{L_{x}^{p}}\right\|_{L_{I}^{2}} \\
& \lesssim T^{\frac{1}{q^{*}}}\left\||x|^{-b}\right\|_{L^{\gamma}(A)}\left\|D^{s_{c}} u\right\|_{L_{I}^{q} L_{x}^{p}}^{2 \sigma}\|\nabla u\|_{L_{I}^{q} L_{x}^{p}} \\
& +T^{\frac{1}{q^{*}}}\left\||x|^{-b-1}\right\|_{L^{d}(A)}\left\|D^{s_{c}} u\right\|_{L_{I}^{q} L_{x}^{p}}^{2 \sigma}\|\nabla u\|_{L_{I}^{q} L_{x}^{p}},
\end{aligned}
$$

if the following conditions are satisfied

$$
\left\{\begin{array}{l}
\frac{1}{p^{*}}=\frac{1}{\gamma}+\frac{1}{\beta}+\frac{1}{p}=\frac{1}{d}+\frac{1}{e}+\frac{1}{f}, \\
s_{c}=\frac{N}{p}-\frac{N}{2 \sigma \beta}=\frac{N}{p}-\frac{N}{2 \sigma e}, \quad 1=\frac{N}{p}-\frac{N}{f}, \quad p<N \\
\frac{1}{2}=\frac{1}{q^{*}}+\frac{2 \sigma}{q}+\frac{1}{q} .
\end{array}\right.
$$

The above conditions are equivalent to

$$
\left\{\begin{array}{cl}
\frac{N}{\gamma}-b & =\frac{N}{p^{*}}+2 \sigma s_{c}-\frac{N(2 \sigma+1)}{p}-b, p<N \\
\frac{N}{d}-b-1 & =\frac{N}{p^{*}}+2 \sigma s_{c}-\frac{N(2 \sigma+1)}{p}-b \\
\frac{1}{q^{*}} & =\frac{1}{2}-\frac{2 \sigma+1}{q} .
\end{array}\right.
$$

Our goal is to find a pair $(q, p) L^{2}$-admissible such that $\left\||x|^{-b}\right\|_{L^{\gamma}(A)}$ and $\left\||x|^{-b-1}\right\|_{L^{d}(A)}$ are bounded quantities (see (3.1)), $p<N$ and $\frac{1}{q^{*}}>0$. Let $\left(q_{ \pm}, p_{ \pm}\right)$defined by

$$
p_{ \pm}=\frac{2 \sigma(2 \sigma+1) N}{2 \sigma^{2} N+2-b \pm \varepsilon} \quad \text { and } \quad q_{ \pm}=\frac{4 \sigma(2 \sigma+1)}{\sigma N-2+b \mp \varepsilon}
$$

for $\varepsilon>0$ sufficiently small. Note that $\frac{2}{q_{ \pm}}=\frac{N}{2}-\frac{N}{p_{ \pm}}$and $p_{ \pm}, q_{ \pm}>0$ whenever $\frac{2-b}{N}<\sigma<\frac{2-b}{N-2}$. Furthermore, $2<p_{ \pm}<\frac{2 N}{N-2}$ and $p_{ \pm}<N$. Indeed, since $\sigma(2 \sigma+1) N>2 \sigma^{2} N+2-b \pm \varepsilon$ and $\varepsilon>0$ small enough, we get $p_{ \pm}>2$. As $-\sigma(N-2)+2-b \pm \varepsilon>0$ for $\varepsilon$ sufficiently small, then $-4 \sigma^{2}<-\sigma(N-2)+2-b \pm \varepsilon$. The last inequality is equivalent to $\sigma(2 \sigma+1)(N-2)<2 \sigma^{2} N+2-b \pm \varepsilon$, which implies $p_{ \pm}<\frac{2 N}{N-2}$. If $N \geqslant 4$, then $N>\frac{2 N}{N-2}>p_{ \pm}$. When $N=3$, we have $p_{ \pm}<3$ if, and only if, $0<2 \sigma^{2}-2 \sigma+2-b \pm \varepsilon$ which is true for $\varepsilon$ small enough and $0<b<\frac{3}{2}$. Summing up,

$$
\left(q_{ \pm}, p_{ \pm}\right) \text {is } L^{2} \text {-admissible and } p_{ \pm}<N \text {. }
$$


In addition,

$$
\frac{1}{q_{ \pm}^{*}}=\frac{1}{2}-\frac{2 \sigma+1}{q_{ \pm}}=\frac{1}{2}-\frac{\sigma-2+b \mp \varepsilon}{4 \sigma}=\frac{-\sigma(N-2)+2-b \pm \varepsilon}{4 \sigma}>0 .
$$

Now, if $A=B^{c}$ we choose $(q, r)=\left(q_{+}, r_{+}\right)$and $\theta_{1}=\frac{1}{q_{+}^{*}}$. Then, $\frac{N}{\gamma}-b<0$ and $\frac{N}{d}-b-1<0$, and consequently, $\left\||x|^{-b}\right\|_{L^{\gamma\left(B^{c}\right)}},\left\||x|^{-b-1}\right\|_{L^{d}\left(B^{c}\right)}<\infty$. On the other hand, if $A=B$ we choose the pair $(q, r)=\left(q_{-}, p_{-}\right)$and $\theta_{2}=\frac{1}{q_{-}^{*}}$, so we also get $\left\||x|^{-b}\right\|_{L^{\gamma}(B)},\left\||x|^{-b-1}\right\|_{L^{d}(B)}<\infty$. Finally, the relations (3.15) and (3.16) imply the desired result.

Lemma 3.4. Let $N \geqslant 3,0<b<2$ and $\frac{2-b}{N}<\sigma<\frac{2-b}{N-2}$, then there exist $c, \theta_{1}, \theta_{2}>0$ and $\theta \in(0,2 \sigma)$ small enough such that the following inequality holds

$$
\begin{gathered}
\left\|\chi_{B^{c}}|x|^{-b}|u|^{2 \sigma} v\right\|_{S^{\prime}\left(\dot{H}^{-s_{c}} ; I\right)}+\left\|\chi_{B}|x|^{-b}|u|^{2 \sigma} v\right\|_{S^{\prime}\left(\dot{H}^{-s_{c}} ; I\right)} \\
\quad \leqslant c\left(T^{\theta_{1}}+T^{\theta_{2}}\right)\|\nabla u\|_{L_{I}^{\infty} L_{x}^{2}}^{\theta}\|u\|_{S\left(\dot{H}^{s_{c}} ; I\right)}^{2 \sigma-\theta}\|v\|_{S\left(\dot{H}^{s_{c}} ; I\right)},
\end{gathered}
$$

where $I=[-T, T]$.

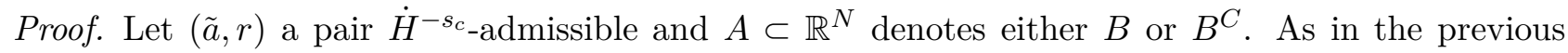
lemmas, an application of the Hölder inequality first in space and then in time yields

$$
\begin{aligned}
& \left\||x|^{-b}|u|^{2 \sigma} v\right\|_{L_{I}^{\tilde{a}^{\prime}} L_{x}^{r^{\prime}(A)}} \leqslant\|\||x|^{-b}\left\|_{L^{\gamma}(A)}\right\| u\left\|_{L_{x}^{\theta r}}^{\theta}\right\| u\left\|_{L_{x}^{r}}^{2 \sigma-\theta}\right\| v\left\|_{L_{x}^{r}}\right\|_{L_{I}^{\tilde{a}^{\prime}}} \\
& \lesssim\left\||x|^{-b}\right\|_{L^{\gamma}(A)}\|\| \nabla u\left\|_{L_{x}^{2}}^{\theta}\right\| u\left\|_{L_{x}^{r}}^{2 \sigma-\theta}\right\| v\left\|_{L_{x}^{r}}\right\|_{L_{I}^{\tilde{a}^{\prime}}} \\
& \lesssim T^{\frac{1}{q_{1}}}\left\||x|^{-b}\right\|_{L^{\gamma}(A)}\|\nabla u\|_{L_{t}^{\infty} L_{x}^{2}}^{\theta}\|u\|_{L_{t}^{a} L_{x}^{r}}^{2 \sigma-\theta}\|v\|_{L_{t}^{a} L_{x}^{r}},
\end{aligned}
$$

assuming the following relations hold

$$
\left\{\begin{aligned}
\frac{1}{r^{\prime}} & =\frac{1}{\gamma}+\frac{1}{r_{1}}+\frac{2 \sigma-\theta}{r}+\frac{1}{r}, \\
1 & =\frac{N}{2}-\frac{N}{\theta r_{1}} \\
\frac{1}{\tilde{a}^{\prime}} & =\frac{1}{q_{1}}+\frac{2 \sigma-\theta}{a}+\frac{1}{a}
\end{aligned}\right.
$$

for $\theta \in(0,2 \sigma)$ small enough.

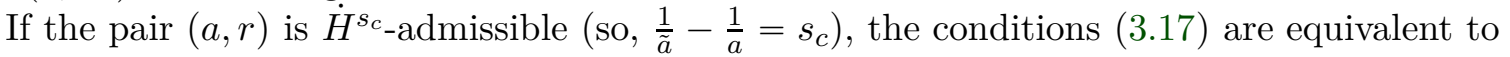

$$
\left\{\begin{aligned}
\frac{N}{\gamma}-b & =N-b-\frac{N \theta}{2}+\theta-\frac{N(2 \sigma+2-\theta)}{r} \\
\frac{1}{q_{1}} & =1-s_{c}-\frac{2 \sigma+2-\theta}{a} .
\end{aligned}\right.
$$

Now, we shall choose $(a, r)$ satisfying (3.18), $\frac{1}{q_{1}}>0$ and $\frac{N}{\gamma}-b>0$, if $A=B$ or $\frac{N}{\gamma}-b<0$, if $A=B^{c}$ (see (3.1) $)^{12}$. We first treat the case $A=B$ and define

$$
a=\frac{2 \sigma+2-\theta}{1-s_{c}-\varepsilon} \quad \text { and } \quad r=\frac{N \sigma_{c}(2 \sigma+2-\theta)}{N(2 \sigma+2-\theta)-2 \sigma_{c}\left(1-s_{c}-\varepsilon\right)},
$$

for $0<\varepsilon<\frac{\theta\left(1-s_{c}\right)}{2}$ small enough. Thus, $(a, r)$ is a $\dot{H}^{s_{c}}$-admissible ${ }^{13}$ and

$$
\begin{aligned}
\frac{1}{q_{1}} & =1-s_{c}-\frac{2 \sigma+2-\theta}{a}=\varepsilon>0, \\
\frac{N}{\gamma}-b & =N-b-\frac{N \theta}{2}+\theta-\frac{N(2 \sigma+2-\theta)}{\sigma_{c}}+2\left(1-s_{c}-\varepsilon\right)=\theta\left(1-s_{c}\right)-2 \varepsilon>0 .
\end{aligned}
$$

\footnotetext{
${ }^{12}$ Note that if we find this pair then we also find a pair $(\tilde{a}, r) \dot{H}^{-s_{c}}$-admissible using the relation $\frac{1}{\tilde{a}}-\frac{1}{a}=s_{c}$.

${ }^{13}$ Since $a>\frac{2}{1-s_{c}}$ and $\frac{2}{a}=\frac{N}{\sigma_{c}}-\frac{N}{r}$, we have $r<\frac{2 N}{N-2}$. On the other hand, $r>\sigma_{c}$ if, and only if, $0<\varepsilon<1-s_{c}$.
} 
Next, we consider $A=B^{c}$ and define the following numbers

$$
a=\infty \quad \text { and } r=\frac{2 N}{N-2 s_{c}}=\frac{2 \sigma N}{2-b} .
$$

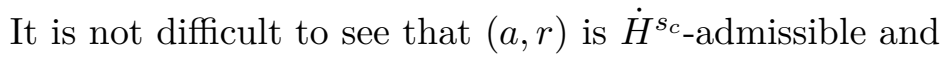

$$
\begin{aligned}
\frac{1}{q_{1}} & =1-s_{c}>0, \\
\frac{N}{\gamma}-b & =N-b-\frac{N \theta}{2}+\theta-\frac{(2 \sigma+2-\theta)(2-b)}{2 \sigma}=-(2-\theta)\left(1-s_{c}\right)<0 .
\end{aligned}
$$

This complete the proof of Lemma 3.4.

Now, with the previous lemmas in hand we are in a position to prove Theorem 1.2.

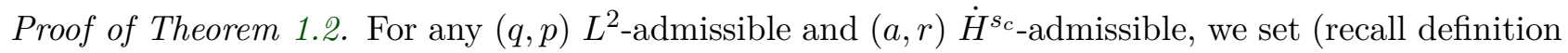
$(2.5))$

$$
X=\left(\bigcap_{(q, p) \in \mathcal{A}_{0}} L^{q}\left([-T, T] ; \dot{H}^{s_{c}, p} \cap \dot{H}^{1, p}\right)\right) \bigcap\left(\bigcap_{(a, r) \in \mathcal{A}_{s_{c}}} L^{a}\left([-T, T] ; L^{r}\right)\right)
$$

and

$$
\|u\|_{T}=\|\nabla u\|_{S\left(L^{2} ; I\right)}+\left\|D^{s_{c}} u\right\|_{S\left(L^{2} ; I\right)}+\|u\|_{S\left(\dot{H}^{\left.s_{c} ; I\right)}\right.},
$$

where $I=[-T, T]$.

For $m, T>0$, define the set

$$
S(m, T)=\left\{u \in X:\|u\|_{T} \leqslant m\right\}
$$

with the metric

$$
d_{T}(u, v)=\|u-v\|_{S\left(\dot{H}^{s} ; I\right)} \cdot
$$

In Appendix A we prove that $\left(S(m, T), d_{T}\right)$ is a complete metric space.

We shall show that $G=G_{u_{0}}$ defined by the right hand side of (1.3) is a contraction on $\left(S(m, T), d_{T}\right)$ for a suitable choice of $m$ and $T$. Indeed, it follows from the Strichartz inequalities in Lemma 2.3 that

$$
\begin{gathered}
\|\nabla G(u)\|_{S\left(L^{2} ; I\right)} \leqslant c\left\|\nabla u_{0}\right\|_{L^{2}}+\left\|\nabla \int_{0}^{t} e^{i\left(t-t^{\prime}\right) \Delta}|x|^{-b}|u|^{2 \sigma} u\left(t^{\prime}\right) d t^{\prime}\right\|_{S\left(L^{2} ; I\right)} \\
\leqslant c\left\|\nabla u_{0}\right\|_{L^{2}}+c\left\|\chi_{B} \nabla|x|^{-b}|u|^{2 \sigma} u\right\|_{S^{\prime}\left(L^{2} ; I\right)}+c\left\|\chi_{B^{c}} \nabla|x|^{-b}|u|^{2 \sigma} u\right\|_{S^{\prime}\left(L^{2} ; I\right)}, \\
\left\|D^{s_{c}} G(u)\right\|_{S\left(L^{2} ; I\right)} \leqslant c\left\|D^{s_{c}} u_{0}\right\|_{L^{2}}+c\left\|D^{s_{c}}|x|^{-b}|u|^{2 \sigma} u\right\|_{S^{\prime}\left(L^{2} ; I\right)},
\end{gathered}
$$

and

$$
\|G(u)\|_{S\left(\dot{H}^{s_{c} ; I}\right)} \leqslant c\left\|u_{0}\right\|_{\dot{H}^{s_{c}}}+c\left\|\chi_{B}|x|^{-b}|u|^{2 \sigma} u\right\|_{S^{\prime}\left(\dot{H}^{-s_{c} ; I}\right)}+c\left\|\chi_{B^{c}}|x|^{-b}|u|^{2 \sigma} u\right\|_{S^{\prime}\left(\dot{H}^{-s_{c} ; I}\right)} .
$$

So, applying Lemmas 3.2-3.4 we deduce

$$
\begin{gathered}
\left\|\chi_{B} \nabla|x|^{-b}|u|^{2 \sigma} u\right\|_{S^{\prime}\left(L^{2} ; I\right)}+\left\|\chi_{B^{c}} \nabla|x|^{-b}|u|^{2 \sigma} u\right\|_{S^{\prime}\left(L^{2} ; I\right)} \leqslant c\left(T^{\theta_{1}}+T^{\theta_{2}}\right)\|\nabla u\|_{S\left(L^{2} ; I\right)}^{2 \sigma+1}, \\
\left\|D^{s_{c}}|x|^{-b}|u|^{2 \sigma} u\right\|_{S^{\prime}\left(L^{2} ; I\right)} \leqslant c\left(T^{\theta_{1}}+T^{\theta_{2}}\right)\|\nabla u\|_{S\left(L^{2} ; I\right)}\left\|D^{s_{c}} u\right\|_{S\left(L^{2} ; I\right)}^{2 \sigma},
\end{gathered}
$$

and

$$
\left\|\chi_{B}|x|^{-b}|u|^{2 \sigma} u\right\|_{S^{\prime}\left(\dot{H}^{-s_{c}} ; I\right)}+\left\|\chi_{B^{c}}|x|^{-b}|u|^{2 \sigma} u\right\|_{S^{\prime}\left(\dot{H}^{-s_{c}} ; I\right)} \leqslant c\left(T^{\theta_{1}}+T^{\theta_{2}}\right)\|\nabla u\|_{L_{t}^{\infty} L_{x}^{2}}^{\theta}\|u\|_{S\left(\dot{H}^{s_{c}} ; I\right)}^{2 \sigma+1-\theta},
$$


for some $\theta_{1}, \theta_{2}>0$. Hence, if $u \in S(m, T)$ then

$$
\|G(u)\|_{T} \leqslant c\left\|u_{0}\right\|_{\dot{H}^{s_{c}} \cap \dot{H}^{1}}+c\left(T^{\theta_{1}}+T^{\theta_{2}}\right) m^{2 \sigma+1} .
$$

Now, choosing $m \geqslant 2 c\left\|u_{0}\right\|_{\dot{H}^{s_{c}} \cap \dot{H}^{1}}$ and $T>0$ such that

$$
c\left(T^{\theta_{1}}+T^{\theta_{2}}\right) m^{2 \sigma}<\frac{1}{4},
$$

we obtain $G(u) \in S(m, T)$. Such calculations establishes that $G$ is well defined on $S(m, T)$. To prove that $G$ is a contraction we first recall the elementary inequality

$$
\left.|| x\right|^{-b}|u|^{2 \sigma} u-\left.|x|^{-b}|v|^{2 \sigma} v|\lesssim| x\right|^{-b}\left(|u|^{2 \sigma}+|v|^{2 \sigma}\right)|u-v| .
$$

Then, an application of Lemma 3.4 yields

$$
\begin{aligned}
d_{T}(G(u), G(v)) & \leqslant c\left(T^{\theta_{1}}+T^{\theta_{2}}\right)\left(\|\nabla u\|_{L_{t}^{\infty} L_{x}^{2}}^{\theta}\|u\|_{S\left(\dot{H}^{s_{c}} ; I\right)}^{2 \sigma-\theta}+\|\nabla v\|_{L_{t}^{\infty \infty} L_{x}^{2}}^{\theta}\|v\|_{S\left(\dot{H}^{s_{c}} ; I\right)}^{2 \sigma-\theta}\right)\|u-v\|_{S\left(\dot{H}^{s_{c}} ; I\right)} \\
& \leqslant c\left(T^{\theta_{1}}+T^{\theta_{2}}\right)\left(\|u\|_{T}^{2 \sigma}+\|v\|_{T}^{2 \sigma}\right) d_{T}(u, v),
\end{aligned}
$$

and so, taking $u, v \in S(m, T)$ we get

$$
d_{T}(G(u), G(v)) \leqslant c\left(T^{\theta_{1}}+T^{\theta_{2}}\right) m^{2 \sigma} d_{T}(u, v) .
$$

Therefore, from (3.20), $G$ is also a contraction on $S(m, T)$. Finally, by the contraction mapping principle we have a unique $u \in S(m, T)$ such that $G(u)=u$ and the proof is completed.

Let $T^{*}=T^{*}\left(u_{0}\right)>0$ be the maximal positive time of existence for a solution $u$ to (1.1) in $\dot{H}^{s_{c}}\left(\mathbb{R}^{N}\right) \cap \dot{H}^{1}\left(\mathbb{R}^{N}\right)$ given by Theorem 1.2. If $T^{*}=\infty$, we say that the solution is global. On the other hand if $T^{*}<\infty$, as consequence of the proof of Theorem 1.2, we get the following blow-up alternative and a lower bound on the blow-up rate.

Corollary 3.5. Let $N \geqslant 3$ and $0<b<\min \left\{\frac{N}{2}, 2\right\}$. If $u$ is a solution to the IVP (1.1) with finite maximal positive time of existence $0<T^{*}<\infty$, then $\lim _{t \rightarrow T^{*}}\|u(t)\|_{\dot{H}^{s} \cap \dot{H}^{1}}=\infty$. Moreover, there exist $c, \widetilde{\theta}_{1}, \widetilde{\theta}_{2}>0$ such that

$$
\|u(t)\|_{\dot{H}^{s_{c}} \cap \dot{H}^{1}}>\frac{c}{\left(T^{*}-t\right)^{\widetilde{\theta}_{1}}+\left(T^{*}-t\right)^{\widetilde{\theta}_{2}}}, \quad \text { for all } \quad t \in\left[0, T^{*}\right) .
$$

Proof. Assume that there exist $0<m<\infty$ and a sequence $\left\{t_{n}\right\}_{n \in \mathbb{N}}$ with $t_{n} \uparrow T^{*}$ such that $\left\|u\left(t_{n}\right)\right\|_{\dot{H}^{s_{c}} \cap \dot{H}^{1}} \leqslant$ $m$ for all $n \geqslant 1$. Let $T(m)$ denote the existence time obtained by Theorem 1.2 for all initial data bounded above by $m$ and $k \in \mathbb{N}$ such that $t_{k}+T(m)>T^{*}$. By (3.20) and starting from $u\left(t_{k}\right)$, one can extend $u$ up to $t_{k}+T(m)$, which contradicts the maximality of $T^{*}$, and thus,

$$
\|u(t)\|_{\dot{H}^{s_{c}} \cap \dot{H}^{1}} \rightarrow \infty, \quad \text { as } t \uparrow T^{*} .
$$

Moreover, it follows from (3.19) and the fixed point argument that if for some $m>0$,

$$
c\|u(t)\|_{\dot{H}^{s_{c}} \cap \dot{H}^{1}}+c\left[(\tau-t)^{\theta_{1}}+(\tau-t)^{\theta_{2}}\right] m^{2 \sigma+1} \leqslant m,
$$

then $\tau<T^{*}$. Thus,

$$
c\|u(t)\|_{\dot{H}^{s} \cap \dot{H}^{1}}+c\left[\left(T^{*}-t\right)^{\theta_{1}}+\left(T^{*}-t\right)^{\theta_{2}}\right] m^{2 \sigma+1}>m,
$$

for all $m>0$. Choosing $m=2 c\|u(t)\|_{\dot{H}^{s_{c}} \cap \dot{H}^{1}}$, it follows that

$$
\left[\left(T^{*}-t\right)^{\theta_{1}}+\left(T^{*}-t\right)^{\theta_{2}}\right]\|u(t)\|_{\dot{H}^{s_{c}} \cap \dot{H}^{1}}^{2 \sigma}>c .
$$

In particular, we have

$$
\|u(t)\|_{\dot{H}^{s_{c}} \cap \dot{H}^{1}}>\frac{c}{\left(T^{*}-t\right)^{\tilde{\theta}_{1}}+\left(T^{*}-t\right)^{\widetilde{\theta}_{2}}},
$$

with $\widetilde{\theta}_{i}=\frac{\theta_{i}}{2 \sigma}$ for $i=1,2$, completing the proof. 
Remark 3.6. It is possible to derive a more precise lower bound on the blow-up rate for type II blowup solutions. Let $u \in C\left(\left[0, T^{*}\right) ; \dot{H}^{s_{c}} \cap \dot{H}^{1}\right)$ be a solution to the IVP (1.1) with finite maximal positive time of existence $0<T^{*}<\infty$. If we assume the condition

$$
\sup _{t \in\left[0, T^{*}\right)}\|u(t)\|_{\dot{H}^{s_{c}}}=M<\infty
$$

then from the local well-posedness theory in $\dot{H}^{s_{c}}\left(\mathbb{R}^{N}\right) \cap \dot{H}^{1}\left(\mathbb{R}^{N}\right)$, we deduce the following lower bound for the blow-up rate

$$
\|\nabla u(t)\|_{L^{2}} \geqslant \frac{c}{\left(T^{*}-t\right)^{\frac{1-s_{c}}{2}}}, \quad \text { for all } t \in\left[0, T^{*}\right)
$$

Indeed, for $t \in\left[0, T^{*}\right)$ we consider the following scaling of $u$

$$
v^{t}(x, \tau)=\rho^{\frac{2-b}{2 \sigma}}(t) u\left(\rho(t) x, t+\rho^{2}(t) \tau\right)
$$

where $\rho(t)^{1-s_{c}}\|\nabla u(t)\|_{L^{2}}=1$. Hence, $v^{t}(0) \in \dot{H}^{s_{c}}\left(\mathbb{R}^{N}\right) \cap \dot{H}^{1}\left(\mathbb{R}^{N}\right)$ and, by simple computations, we can find $m>0$, such that $\left\|v^{t}(0)\right\|_{\dot{H}^{s_{c}} \cap \dot{H}^{1}} \leqslant m$ for all $t \in\left[0, T^{*}\right)$. Thus, from the local theory in $\dot{H}^{s_{c}}\left(\mathbb{R}^{N}\right) \cap$ $\dot{H}^{1}\left(\mathbb{R}^{N}\right)$, there exists $\tau_{0}$, independent of $t$, such that $v^{t}$ is defined on $\left[0, \tau_{0}\right]$. Then, $t+\rho^{2}(t) \tau_{0}<T^{*}$, and consequently we obtain (3.21).

\section{Gagliardo-Nirenberg inequality and Global solutions}

As we mentioned in the introduction, using a Sobolev embedding (see Stein-Weiss [33, Theorem $\left.\mathrm{B}^{*}\right]$ ), Campos and the first author [4] established the following Gagliardo-Nirenberg type inequality for functions $f \in \dot{H}^{1}\left(\mathbb{R}^{N}\right) \cap L^{\sigma_{c}}\left(\mathbb{R}^{N}\right)$

$$
\int|x|^{-b}|f|^{2 \sigma+2} d x \leqslant c\|\nabla f\|_{L^{2}}^{2}\|f\|_{L^{\sigma_{c}}}^{2 \sigma}
$$

where $N \geqslant 2,0<b<2, \sigma$ in the intercritical regime $\left(\frac{2-b}{N}<\sigma<\frac{2-b}{N-2}\right.$, if $N \geqslant 3$ or $\frac{2-b}{N}<\sigma<\infty$, if $N=2$ ) and $\sigma_{c}=\frac{2 N \sigma}{2-b}$. In this section, we investigate the sharp constant for inequality above. As a consequence we also prove that this inequality holds when $N=1$ (with the same restrictions on the other parameters).

\subsection{The ground states}

We first recall that for $N \geqslant 1,0<b<2$ and $\sigma$ in the intercritical regime the second author [12], following the ideas introduced by Weinstein [37], obtained the following Gagliardo-Nirenberg type inequality

$$
\int|x|^{-b}|f|^{2 \sigma+2} d x \leqslant C_{G N}\|\nabla f\|_{L^{2}}^{2 \sigma s_{c}+2}\|f\|_{L^{2}}^{2 \sigma\left(1-s_{c}\right)}
$$

with the sharp constant $C_{G N}>0$ given explicitly by

$$
C_{G N}=\left[\frac{2 \sigma\left(1-s_{c}\right)}{2 \sigma s_{c}+2}\right]^{\sigma s_{c}} \frac{2 \sigma+2}{\left(2 \sigma s_{c}+2\right)\|Q\|_{L^{2}}^{2 \sigma}},
$$

where $Q$ is the unique radially-symmetric, positive, decreasing solution of the elliptic problem

$$
\Delta Q+|x|^{-b}|Q|^{2 \sigma} Q=Q .
$$

The proof relies mainly on the fact that the functional $f \mapsto \int|x|^{-b}|f|^{2 \sigma+2}$ is weakly sequentially continuously (see Genoud [17, Lemma 2.1] and the references therein), Here we follow a similar approach 
to study the inequality (4.1) and, as we will see below, the sharp constant is directly connected with the solutions of the elliptic equation

$$
\Delta \phi+|x|^{-b}|\phi|^{2 \sigma} \phi=|\phi|^{\sigma_{c}-2} \phi .
$$

In the following lemma we obtain two Pohozaev-type identities which are satisfied by any solution of $(4.2)$.

Lemma 4.1. Let $N \geqslant 1,0<b<2, \frac{2-b}{N}<\sigma<\frac{2-b}{N-2}\left(\frac{2-b}{N}<\sigma<\infty\right.$, if $\left.N=1,2\right)$ and $\sigma_{c}=\frac{2 N \sigma}{2-b}$. Let $\phi \in \dot{H}^{1}\left(\mathbb{R}^{N}\right) \cap L^{\sigma_{c}}\left(\mathbb{R}^{N}\right)$ be a solution of (4.2). Then the following identities hold

$$
\begin{gathered}
\int|\nabla \phi|^{2} d x=\frac{1}{\sigma} \int|\phi|^{\sigma_{c}} d x \\
\int|x|^{-b}|\phi|^{2 \sigma+2} d x=\frac{\sigma+1}{\sigma} \int|\phi|^{\sigma_{c}} d x .
\end{gathered}
$$

Proof. The proof of these identities is classical and we provide the details for the reader's convenience. Multiplying the equation (4.2) by $x \cdot \nabla \bar{\phi}$ and taking the real part, we obtain

$$
\operatorname{Re} \int \Delta \phi x \cdot \nabla \bar{\phi} d x+\operatorname{Re} \int|x|^{-b}|\phi|^{2 \sigma} \phi x \cdot \nabla \bar{\phi} d x=\operatorname{Re} \int|\phi|^{\sigma_{c}-2} \phi x \cdot \nabla \bar{\phi} d x .
$$

We consider the first term in the left hand side of (4.5). Indeed, integrating by parts, we get

$$
\begin{aligned}
\int \Delta \phi x \cdot \nabla \bar{\phi} d x & =\sum_{i, j=1}^{N} \int \partial_{i}^{2} \phi x_{j} \partial_{j} \bar{\phi} d x=-\sum_{i, j=1}^{N} \int \partial_{i} \phi \partial_{i}\left(x_{j} \partial_{j} \bar{\phi}\right) d x \\
& =-\sum_{i=1}^{N} \int \partial_{i} \phi \partial_{i} \bar{\phi} d x-\sum_{i, j=1}^{N} \int \partial_{i} \phi x_{j} \partial_{i j}^{2} \bar{\phi} d x \\
& =-\int|\nabla \phi|^{2} d x-\sum_{i, j=1}^{N} \int \phi\left(\delta_{i j} \partial_{i j}^{2} \bar{\phi}+x_{j} \partial_{i i j}^{3} \bar{\phi}\right) d x \\
& =-2 \int|\nabla \phi|^{2} d x-\sum_{i, j=1}^{N} \int \phi x_{j} \partial_{i i j}^{3} \bar{\phi} d x \\
& =-2 \int|\nabla \phi|^{2} d x-\sum_{i, j=1}^{N} \int\left(\partial_{j} \phi x_{j}+\phi\right) \partial_{i i}^{2} \bar{\phi} d x \\
& =-2 \int|\nabla \phi|^{2} d x-\int \Delta \phi x \cdot \nabla \bar{\phi} d x+N \int|\nabla \phi|^{2} d x,
\end{aligned}
$$

and thus,

$$
\operatorname{Re} \int \Delta \phi x \cdot \nabla \bar{\phi} d x=\left(\frac{N}{2}-1\right) \int|\nabla \phi|^{2} d x
$$

For the second term in the left hand side of (4.5), we also integrate by parts to deduce

$$
\begin{aligned}
\int|x|^{-b}|\phi|^{2 \sigma} \phi x \cdot \nabla \phi d x= & \sum_{j=1}^{N} \int|x|^{-b}|\phi|^{2 \sigma} \phi x_{j} \partial_{j} \bar{\phi} d x=-\sum_{j=1}^{N} \int \partial_{j}\left(|x|^{-b}|\phi|^{2 \sigma} \phi x_{j}\right) \bar{\phi} d x \\
= & b \int|x|^{-b}|\phi|^{2 \sigma+2} d x-2 \sigma \operatorname{Re} \int|x|^{-b}|\phi|^{2 \sigma} \bar{\phi} x \cdot \nabla \phi d x \\
& -N \int|x|^{-b}|\phi|^{2 \sigma+2} d x-\int|x|^{-b}|\phi|^{2 \sigma} \bar{\phi} x \cdot \nabla \phi d x
\end{aligned}
$$


which implies

$$
\operatorname{Re} \int|x|^{-b}|\phi|^{2 \sigma} \phi x \cdot \nabla \bar{\phi} d x=-\frac{N-b}{2 \sigma+2} \int|x|^{-b}|\phi|^{2 \sigma+2} d x .
$$

The term in the right hand side of (4.5) can be treated in the same way to obtain

$$
\operatorname{Re} \int|\phi|^{\sigma_{c}-2} \phi x \cdot \nabla \bar{\phi} d x=-\frac{2-b}{2 \sigma} \int|\phi|^{\sigma_{c}} d x .
$$

From (4.5), (4.6), (4.7) and (4.8), we get

$$
\frac{N-b}{2 \sigma+2} \int|x|^{-b}|\phi|^{2 \sigma+2} d x=\left(\frac{N}{2}-1\right) \int|\nabla \phi|^{2} d x+\frac{2-b}{2 \sigma} \int|\phi|^{\sigma_{c}} d x .
$$

Now, multiplying (4.2) by $\phi$ and integrating, we obtain

$$
\int|x|^{-b}|\phi|^{2 \sigma+2} d x=\int|\nabla \phi|^{2} d x+\int|\phi|^{\sigma_{c}} d x .
$$

From the identities (4.9) and (4.10), it is easy to deduce the relations (4.3) and (4.4).

Now, consider the functional space

$$
L_{b}^{2 \sigma+2}\left(\mathbb{R}^{N}\right)=\left\{f \in \mathcal{M}\left(\mathbb{R}^{N} ; \mathbb{C}\right) ; \quad \int|x|^{-b}|f|^{2 \sigma+2} d x<\infty\right\}
$$

where $\mathcal{M}\left(\mathbb{R}^{N} ; \mathbb{C}\right)$ denotes the set of all measurable function $f: \mathbb{R}^{N} \rightarrow \mathbb{C}$. In this space we define the norm

$$
\|f\|_{L_{b}^{2 \sigma+2}}=\left(\int|x|^{-b}|f|^{2 \sigma+2} d x\right)^{\frac{1}{2 \sigma+2}} .
$$

In the next proposition we prove an useful compact embedding.

Proposition 4.2. Let $N \geqslant 1,0<b<2, \frac{2-b}{N}<\sigma<\frac{2-b}{N-2}\left(\frac{2-b}{N}<\sigma<\infty\right.$, if $\left.N=1,2\right)$ and $\sigma_{c}=\frac{2 N \sigma}{2-b}$. Then, the embedding

$$
\dot{H}^{1}\left(\mathbb{R}^{N}\right) \cap L^{\sigma_{c}}\left(\mathbb{R}^{N}\right) \subset L_{b}^{2 \sigma+2}\left(\mathbb{R}^{N}\right)
$$

is compact.

Proof. Let $\left\{f_{n}\right\}_{n \in \mathbb{N}}$ be a bounded sequence in $\dot{H}^{1}\left(\mathbb{R}^{N}\right) \cap L^{\sigma_{c}}\left(\mathbb{R}^{N}\right)$. Then, there exists $f \in \dot{H}^{1}\left(\mathbb{R}^{N}\right) \cap$ $L^{\sigma_{c}}\left(\mathbb{R}^{N}\right)$ such that, up to a subsequence, $f_{n} \rightarrow f$ in $\dot{H}^{1}\left(\mathbb{R}^{N}\right) \cap L^{\sigma_{c}}\left(\mathbb{R}^{N}\right)$, as $n \rightarrow \infty$. Defining $w_{n}=f_{n}-f$, we will show that

$$
\int|x|^{-b}\left|w_{n}\right|^{2 \sigma+2} d x \rightarrow 0, \quad \text { as } \quad n \rightarrow \infty .
$$

First, since $\left\{w_{n}\right\}_{n \in \mathbb{N}}$ is uniformly bounded in $\dot{H}^{1}\left(\mathbb{R}^{N}\right) \cap L^{\sigma_{c}}\left(\mathbb{R}^{N}\right)$, from the Gagliardo-Nirenberg inequality $^{14}(2.2)$, we get that

$$
\left\{w_{n}\right\}_{n \in \mathbb{N}} \text { is uniformly bounded in } L^{p}\left(\mathbb{R}^{N}\right) \text { for all } p \in\left(\sigma_{c}, 2^{*}\right),
$$

where

$$
2^{*}=\left\{\begin{array}{l}
\frac{2 N}{N-2}, \quad N \geqslant 3 \\
\infty, \quad N=1,2
\end{array}\right.
$$

\footnotetext{
${ }^{14}$ For $N \geqslant 3$ it is enough to use the Sobolev embedding (2.1) and interpolation.
} 
Moreover, given $\varepsilon>0$ for $R \geqslant \varepsilon^{-\frac{1}{b}}$ we have from (4.11) that

$$
\int_{\mathbb{R}^{N} \backslash B(0, R)}|x|^{-b}\left|w_{n}\right|^{2 \sigma+2} d x \leqslant \varepsilon \int_{\mathbb{R}^{N} \backslash B(0, R)}\left|w_{n}\right|^{2 \sigma+2} \leqslant C \varepsilon,
$$

where $B(0, R)=\left\{x \in \mathbb{R}^{N} ;|x| \leqslant R\right\}$.

Now, we estimate the integral over the ball $B(0, R)$. Note that

$$
\int_{B(0, R)}\left|\nabla w_{n}\right|^{2} d x \leqslant \int\left|\nabla w_{n}\right|^{2} d x .
$$

Moreover, by Hölder's inequality and Sobolev embedding we get for $p \in\left(2,2^{*}\right)$

$$
\int_{B(0, R)}\left|w_{n}\right|^{2} d x \leqslant C_{R, N}\left\|w_{n}\right\|_{L^{p}}^{2} \leqslant C_{R, N}\left\|\nabla w_{n}\right\|_{L^{2}}^{2}
$$

and therefore $\left\{w_{n}\right\}_{n \in \mathbb{N}}$ is uniformly bounded in $H^{1}(B(0, R))$.

From the compact embedding on bounded domains $H^{1}(B(0, R)) \subset L^{p}(B(0, R))$ for $p \in\left(2,2^{*}\right)$ (see, for instance, Cazenave [5, Theorem 1.3.4 $]^{15}$ ) and the fact that $w_{n} \rightarrow 0$ in $L^{\sigma_{c}}\left(\mathbb{R}^{N}\right)$, we deduce that

$$
w_{n} \rightarrow 0 \text { in } L^{p}(B(0, R)) \text { for all } p \in\left(2,2^{*}\right),
$$

up to a subsequence. Again, since $\sigma<\frac{2-b}{N-2}$, we obtain $\frac{N-b}{N}-(\sigma+1) \frac{N-2}{N}>0$. Thus, we can choose $\gamma_{2}^{\prime} \geqslant 1$ such that $\gamma_{2}^{\prime}<\frac{N}{b}$ and $2<(2 \sigma+2) \gamma_{2}<2^{*}$ (where $\gamma_{2}^{\prime}$ is such that $\left.\frac{1}{\gamma_{2}}+\frac{1}{\gamma_{2}^{\prime}}=1\right)$. Hence, by Hölder's inequality we have

$$
\begin{aligned}
\int_{B(0, R)}|x|^{-b}\left|w_{n}\right|^{2 \sigma+2} d x & \leqslant\left(\int_{B(0, R)}|x|^{-b \gamma_{2}^{\prime}} d x\right)^{\frac{1}{\gamma_{2}^{\prime}}}\left(\int_{B(0, R)}\left|w_{n}\right|^{(2 \sigma+2) \gamma_{2}}\right)^{\frac{1}{\gamma_{2}}} \\
& \leqslant\left(\int_{B(0, R)}|x|^{-b \gamma_{2}^{\prime}} d x\right)^{\frac{1}{\gamma_{2}^{\prime}}}\left(\int_{B(0, R)}\left|w_{n}\right|^{(2 \sigma+2) \gamma_{2}} d x\right)^{\frac{1}{\gamma_{2}}}
\end{aligned}
$$

So, in view of (4.12), given $\varepsilon>0$ there exists $n_{0}$ such that for any $n \geqslant n_{0}$

$$
\int_{B(0, R)}|x|^{-b}\left|w_{n}\right|^{2 \sigma+2} d x<\varepsilon
$$

which completes the proof of Proposition 4.2.

We now show Theorem 1.3, which characterizes the sharp constant for the Gagliardo-Nirenberg type inequality (4.1).

Proof of Theorem 1.3. Given $f \in \dot{H}^{1}\left(\mathbb{R}^{N}\right) \cap L^{\sigma_{c}}\left(\mathbb{R}^{N}\right)$, define the Weinstein functional

$$
J(f)=\frac{\|\nabla f\|_{L^{2}}^{2}\|f\|_{L^{\sigma \sigma_{c}}}^{2 \sigma}}{\left\||\cdot|^{\frac{-b}{2 \sigma+2}} f\right\|_{L^{2 \sigma+2}}^{2 \sigma+2}} .
$$

We set $J=\inf _{f \in \dot{H}^{1} \cap L^{\sigma_{c}}, f \neq 0} J(f)$ and consider a minimizing sequence $\left\{f_{n}\right\}_{n \in \mathbb{N}}$. From (4.1), we can deduce that $J>0$. Now rescale $\left\{f_{n}\right\}_{n \in \mathbb{N}}$ by setting

$$
g_{n}(x)=\mu_{n} f_{n}\left(\theta_{n} x\right)
$$

\footnotetext{
${ }^{15}$ In the case $N=1$ we have $H^{1}(B(0, R)) \subset L^{\infty}(B(0, R))$. Since the embedding $L^{\infty}(B(0, R)) \subset L^{p}(B(0, R))$ for all $p \geqslant 1$ is continuous, we also have that the embedding $H^{1}(B(0, R)) \subset L^{p}(B(0, R))$, for all $p \geqslant 1$ is compact.
} 
with

$$
\mu_{n}=\frac{\left\|f_{n}\right\|_{L^{\sigma_{c}}}^{\frac{N-2}{2\left(1-s_{c}\right)}}}{\left\|\nabla f_{n}\right\|_{L^{2}}^{\frac{2-b}{2 \sigma\left(1-s_{c}\right)}}} \quad \text { and } \quad \theta_{n}=\left(\frac{\left\|f_{n}\right\|_{L^{\sigma_{c}}}}{\left\|\nabla f_{n}\right\|_{L^{2}}}\right)^{\frac{1}{1-s_{c}}}
$$

A direct calculation implies

$$
\left\|g_{n}\right\|_{L^{\sigma_{c}}}^{\sigma_{c}}=\mu_{n}^{\sigma_{c}} \int\left|f_{n}\left(\theta_{n} x\right)\right|^{\sigma_{c}} d x=\frac{\mu_{n}^{\sigma_{c}}}{\theta_{n}^{N}}\left\|f_{n}\right\|_{L^{\sigma_{c}}}^{\sigma_{c}}=1
$$

and

$$
\left\|\nabla g_{n}\right\|_{L^{2}}^{2}=\mu_{n}^{2} \int \theta_{n}^{2}\left|\nabla f_{n}\left(\theta_{n} x\right)\right|^{2} d x=\frac{\mu_{n}^{2}}{\theta_{n}^{N-2}}\left\|\nabla f_{n}\right\|_{L^{2}}^{2}=1,
$$

that is, $\left\|g_{n}\right\|_{L^{\sigma_{c}}}=\left\|\nabla g_{n}\right\|_{L^{2}}=1$. Moreover, since $J$ is invariant under this scaling, $\left\{g_{n}\right\}_{n \in \mathbb{N}}$ is also a minimizing sequence and bounded in $\dot{H}^{1}\left(\mathbb{R}^{N}\right) \cap L^{\sigma_{c}}\left(\mathbb{R}^{N}\right)$. Furthermore, there exists $g^{*} \in \dot{H}^{1}\left(\mathbb{R}^{N}\right) \cap$ $L^{\sigma_{c}}\left(\mathbb{R}^{N}\right)$ such that, up to a subsequence, $g_{n} \rightarrow g^{*}$ weakly in $\dot{H}^{1}\left(\mathbb{R}^{N}\right) \cap L^{\sigma_{c}}\left(\mathbb{R}^{N}\right)$, and then

$$
\left\|g^{*}\right\|_{L^{\sigma_{c}}} \leqslant 1 \quad \text { and } \quad\left\|\nabla g^{*}\right\|_{L^{2}} \leqslant 1 .
$$

Thus, from Proposition 4.2, we have

$$
J \leqslant J\left(g^{*}\right) \leqslant \frac{1}{\left\||\cdot|^{\frac{-b}{2 \sigma+2}} g^{*}\right\|_{L^{2 \sigma+2}}^{2 \sigma+2}}=\lim _{n \rightarrow \infty} \frac{1}{\left\|\left.|\cdot|\right|^{\frac{-b}{2 \sigma+2}} g_{n}\right\|_{L^{2 \sigma+2}}^{2 \sigma+2}}=J .
$$

Consequently,

$$
J\left(g^{*}\right)=\frac{1}{\left\|\mid \cdot \frac{\left.\right|^{\frac{-b}{2 \sigma+2}}}{2 \sigma}\right\|_{L^{2 \sigma+2}}^{2 \sigma+2}} \quad \text { and } \quad\left\|g^{*}\right\|_{L^{\sigma_{c}}}=\left\|\nabla g^{*}\right\|_{L^{2}}=1 .
$$

In particular, $g^{*} \neq 0$ and is a minimizer for the functional $J$. Moreover, $g^{*}$ is a solution for the Euler-Lagrange equation

$$
\left.\frac{d}{d s}\right|_{s=0} J\left(g_{s}\right)=0
$$

where $g_{s}=g^{*}+s \varphi$ for $\varphi \in \mathcal{S}\left(\mathbb{R}^{N}\right)$. On the other hand, computing the Fréchet derivative, we deduce

$$
\begin{gathered}
\left.\frac{d}{d s}\right|_{s=0}\left\||\cdot|^{-\frac{b}{2 \sigma+2}} g_{s}\right\|_{L^{2 \sigma+2}}^{2 \sigma+2}=(2 \sigma+2) \operatorname{Re}\left\langle|x|^{-b}\left|g^{*}\right|^{2 \sigma} g^{*}, \varphi\right\rangle, \\
\left.\frac{d}{d s}\right|_{s=0}\left\|\nabla g_{s}\right\|_{L^{2}}^{2}=2 \operatorname{Re}\left\langle\nabla g^{*}, \nabla \varphi\right\rangle=2 \operatorname{Re}\left\langle-\Delta g^{*}, \varphi\right\rangle
\end{gathered}
$$

and

$$
\left.\frac{d}{d s}\right|_{s=0}\left\|g_{s}\right\|_{L^{\sigma_{c}}}^{2 \sigma}=\left.\frac{2 \sigma}{\sigma_{c}}\left\|g^{*}\right\|_{L^{\sigma_{c}}}^{2 \sigma-\sigma_{c}} \frac{d}{d s}\right|_{s=0}\left\|g_{s}\right\|_{L^{\sigma_{c}}}^{\sigma_{c}}=2 \sigma\left\|g^{*}\right\|_{L^{\sigma_{c}}}^{2 \sigma-\sigma_{c}} \operatorname{Re}\left\langle\left.\left|g^{*}\right|\right|^{\sigma_{c}-2} g^{*}, \varphi\right\rangle .
$$

From (4.13), $\left\|v^{*}\right\|_{L^{\sigma_{c}}}=\left\|\nabla v^{*}\right\|_{L^{2}}^{2}=1$ and

$$
J(f)\left\||\cdot|^{-\frac{b}{2 \sigma+2}} f\right\|_{L^{2 \sigma+2}}^{2 \sigma+2}=\|\nabla f\|_{L^{2}}^{2}\|f\|_{L^{\sigma_{C}}}^{2 \sigma}
$$

we have

$$
\operatorname{Re}\left\langle-\Delta g^{*}-(\sigma+1) J|x|^{-b}\left|g^{*}\right|^{2 \sigma} g^{*}+\sigma\left|g^{*}\right| \sigma_{c}-2 g^{*}, \varphi\right\rangle=0
$$


where $\langle\cdot, \cdot\rangle$ is the inner product in $L^{2}$. Taking $i \varphi$ instead of $\varphi$ in (4.14) and using that $\operatorname{Re}(i z)=$ $-\operatorname{Im}(z)$, we get

$$
\operatorname{Im}\left\langle-\Delta v^{*}-(\sigma+1) J|x|^{-b}\left|v^{*}\right|^{2 \sigma} v^{*}+\sigma\left|v^{*}\right| \sigma^{\sigma_{c}-2} v^{*}, \varphi\right\rangle=0 .
$$

Consequently, $g^{*}$ is a solution for elliptic equation

$$
\Delta g^{*}+(\sigma+1) J|x|^{-b}\left|g^{*}\right|^{2 \sigma} g^{*}=\sigma\left|g^{*}\right|^{\sigma_{c}-2} g^{*} .
$$

Now, we take $V$ defined by $g^{*}(x)=\alpha V(\beta x)$ with

$$
\alpha=\left[\frac{(\sigma+1) J}{\sigma^{\frac{2-b}{2}}}\right]^{\frac{2 N}{(2-b)\left[N\left(\sigma_{c}-2\right)-2 \sigma_{c}\right]}} \quad \text { and } \quad \beta=\sigma^{\frac{1}{2}}\left[\frac{(\sigma+1) J}{\sigma^{\frac{2-b}{2}}}\right]^{\frac{N\left(\sigma_{c}-2\right)}{(2-b)\left[N\left(\sigma_{c}-2\right)-2 \sigma_{C}\right]}}
$$

so that $V$ is a solution of $(1.5)$ and

$$
\|V\|_{L^{\sigma_{c}}}^{\sigma_{c}}=\frac{\beta^{N}}{\alpha^{\sigma_{c}}}\left\|g^{*}\right\|_{L^{\sigma_{c}}}^{\sigma_{c}}=\frac{\beta^{N}}{\alpha^{\sigma_{c}}}=[(\sigma+1) J]^{\frac{N}{2-b}}
$$

Note that, this implies

$$
J=\frac{\|V\|_{L^{\sigma_{c}}}^{2 \sigma}}{\sigma+1}
$$

and by the definition of $J$, we have for all $f \in \dot{H}^{1}\left(\mathbb{R}^{N}\right) \cap L^{\sigma_{c}}\left(\mathbb{R}^{N}\right)$

$$
\frac{\|V\|_{L^{\sigma_{c}}}^{2 \sigma}}{\sigma+1}=J \leqslant \frac{\|\nabla f\|_{L^{2}}^{2}\|f\|_{L^{\sigma_{c}}}^{2 \sigma}}{\left\||\cdot| \frac{-b}{2 \sigma+2} f\right\|_{L^{2 \sigma+2}}^{2 \sigma+2}}
$$

which implies (1.4). On the other hand, by Lemma 4.1, if $\phi$ is a solution of the elliptic equation (4.2), then $J(\phi)$ is given by

$$
J(\phi)=\frac{\|\nabla \phi\|_{L^{2}}^{2}\|\phi\|_{L^{\sigma_{c}}}^{2 \sigma}}{\left\||\cdot|^{-\frac{b}{2 \sigma+2}} \phi\right\|_{L^{2 \sigma+2}}^{2 \sigma+2}}=\frac{\frac{1}{\sigma}\|\phi\|_{L^{\sigma_{c}}}^{2 \sigma+\sigma_{c}}}{\frac{\sigma+1}{\sigma}\|\phi\|_{L^{\sigma_{c}}}^{\sigma_{c}}}=\frac{\|\phi\|_{L^{\sigma_{c}}}^{2 \sigma}}{\sigma+1} .
$$

Therefore, since $J(V)=\min _{f \in \dot{H}^{1} \cap L^{\sigma_{c}}, f \neq 0} J(f)$, we have that $V$ is a solution of (1.5) with minimal $L^{\sigma_{c}}\left(\mathbb{R}^{N}\right)$ norm.

As a consequence of the Theorem 1.3, we obtain the following global well-posedness result.

Proof of Theorem 1.4. By the Gagliardo-Nirenberg inequality in Theorem 1.3 and energy conservation, we get

$$
\begin{aligned}
E\left(u_{0}\right)=E(u(t)) & =\frac{1}{2}\|\nabla u(t)\|_{L^{2}}^{2}-\frac{1}{2 \sigma+2}\left\|\left.\cdot \cdot\right|^{-\frac{b}{2 \sigma+2}} u(t)\right\|_{L^{2 \sigma+2}}^{2 \sigma+2} \\
& \geqslant \frac{1}{2}\|\nabla u(t)\|_{L^{2}}^{2}\left(1-\frac{\|u(t)\|_{L^{\sigma_{C}}}^{2 \sigma}}{\|V\|_{L^{\sigma_{c}}}^{2 \sigma}}\right) .
\end{aligned}
$$

Since $\dot{H}^{s_{c}}\left(\mathbb{R}^{N}\right) \subset L^{\sigma_{c}}\left(\mathbb{R}^{N}\right)$, from the assumption $\sup _{t}\|u(t)\|_{\dot{H}^{s_{c}}}<\|V\|_{L^{\sigma_{c}}}$, we deduce that $\|\nabla u(t)\|_{L^{2}}$ is bounded for all $t \in\left[0, T^{*}\right)$. On the other hand, since $\|u(t)\|_{\dot{H}^{s_{c}} \cap \dot{H}^{1}}=\|u(t)\|_{\dot{H}^{s_{c}}}+\|u(t)\|_{\dot{H}^{1}}$, by the blowup alternative (see Corollary 3.5) if $T^{*}<\infty$, then $\lim _{t \uparrow T^{*}}\|\nabla u(t)\|_{L^{2}}=\infty$, which is a contradiction. Consequently, $u$ is a global solution for (1.1).

Remark 4.3. It is possible to replace the assumption $\sup _{t \in\left[0, T^{*}\right)}\|u(t)\|_{\dot{H}^{s_{c}}}<\|V\|_{L^{\sigma_{c}}}$ in the statement of Theorem 1.4 by $\sup _{t \in\left[0, T^{*}\right)}\|u(t)\|_{L^{\sigma_{c}}}<\|V\|_{L^{\sigma_{c}}}$ and (1.6). Indeed, from $\sup _{t \in\left[0, T^{*}\right)}\|u(t)\|_{L^{\sigma_{c}}}<\|V\|_{L^{\sigma_{c}}}$ we deduce that $\|\nabla u(t)\|_{L^{2}}$ is bounded for all $t \in\left[0, T^{*}\right)$. Moreover, the assumption (1.6) and Corollary 3.5 implies $\lim _{t \uparrow T^{*}}\|\nabla u(t)\|_{L^{2}}=\infty$, achieving the contradiction. 


\section{Critical norm concentration}

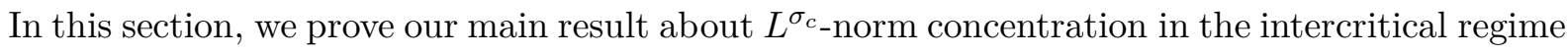
for finite time blow-up solution.

Proof of Theorem 1.5. Let $\left\{t_{n}\right\}_{n \in \mathbb{N}}$ be an arbitrary time sequence such that $t_{n} \uparrow T^{*}$, as $n \rightarrow \infty$. Define

$$
\rho_{n}=\left(\frac{1}{\left\|\nabla u\left(t_{n}\right)\right\|_{L^{2}}}\right)^{\frac{1}{1-s_{c}}} \quad \text { and } \quad v_{n}(x)=\rho_{n}^{\frac{2-b}{2 \sigma}} u\left(\rho_{n} x, t_{n}\right) .
$$

In this case, for all $n \in \mathbb{N}$, we get

$$
\begin{gathered}
\left\|v_{n}\right\|_{\dot{H}^{s_{c}}}=\left\|u\left(t_{n}\right)\right\|_{\dot{H}^{s_{c}}}<\infty, \\
\left\|\nabla v_{n}\right\|_{L^{2}}^{2}=\int\left|\nabla v_{n}\right|^{2} d x=\rho_{n^{\frac{2(2-b)}{2 \sigma}}+2-N}\left\|\nabla u\left(t_{n}\right)\right\|_{L^{2}}^{2}=\rho_{n}^{2\left(1-s_{c}\right)}\left\|\nabla u\left(t_{n}\right)\right\|_{L^{2}}^{2}=1
\end{gathered}
$$

and

$$
E\left(v_{n}\right)=\frac{1}{2}\left\|\nabla v_{n}\right\|_{L^{2}}^{2}-\frac{1}{2 \sigma+2} \int|x|^{-b}\left|v_{n}\right|^{2 \sigma+2} d x=\rho_{n}^{2\left(1-s_{c}\right)} E\left(u_{0}\right) .
$$

So $\left\{v_{n}\right\}_{n \in \mathbb{N}}$ is a bounded sequence in $\dot{H}^{s_{c}}\left(\mathbb{R}^{N}\right) \cap \dot{H}^{1}\left(\mathbb{R}^{N}\right)$ and since $\rho_{n} \rightarrow 0$ when $n \rightarrow \infty$, we have

$$
\lim _{n \rightarrow \infty} E\left(v_{n}\right)=0 .
$$

Thus, there exists $v^{*} \in \dot{H}^{s_{c}}\left(\mathbb{R}^{N}\right) \cap \dot{H}^{1}\left(\mathbb{R}^{N}\right)$ such that, up to a subsequence, $v_{n} \rightarrow v^{*}$ in $\dot{H}^{s_{c}}\left(\mathbb{R}^{N}\right) \cap$ $\dot{H}^{1}\left(\mathbb{R}^{N}\right)$, as $n \rightarrow \infty$. Moreover since $\dot{H}^{s_{c}}\left(\mathbb{R}^{N}\right) \subset L^{\sigma_{c}}\left(\mathbb{R}^{N}\right)$ we have

$$
\left\|\nabla v^{*}\right\|_{L^{2}} \leqslant \liminf _{n \rightarrow \infty}\left\|\nabla v_{n}\right\|_{L^{2}} \quad \text { and } \quad\left\|v^{*}\right\|_{L^{\sigma_{c}}} \leqslant \liminf _{n \rightarrow \infty}\left\|v_{n}\right\|_{L^{\sigma_{c}}} .
$$

In addition, by Proposition 4.2

$$
\lim _{n \rightarrow \infty}\left\||\cdot|^{-\frac{b}{2 \sigma+2}} v_{n}\right\|_{L^{2 \sigma+2}}^{2 \sigma+2}=\left\||\cdot|^{-\frac{b}{2 \sigma+2}} v^{*}\right\|_{L^{2 \sigma+2}}^{2 \sigma+2} .
$$

Hence, from the sharp Gagliardo-Nirenberg inequality in Theorem 1.3, (5.1) and (5.2), we get

$$
0=\liminf _{n \rightarrow \infty} E\left(v_{n}\right) \geqslant \frac{1}{2}\left\|\nabla v^{*}\right\|_{L^{2}}^{2}\left(1-\frac{\left\|v^{*}\right\|_{L^{\sigma_{c}}}^{2 \sigma}}{\|V\|_{L^{\sigma_{c}}}}\right),
$$

which implies $\left\|v^{*}\right\|_{L^{\sigma_{c}}} \geqslant\|V\|_{L^{\sigma_{c}}}$. Consequently, for every $R>0$,

$$
\begin{aligned}
\liminf _{n \rightarrow \infty} \int_{|y| \leqslant \rho_{n} R}\left|u\left(y, t_{n}\right)\right|^{\sigma_{c}} d y & =\liminf _{n \rightarrow \infty} \int_{|x| \leqslant R} \rho_{n}^{\frac{\sigma_{c}(2-b)}{2 \sigma}}\left|u\left(\rho_{n} x, t_{n}\right)\right|^{\sigma_{c}} d x \\
& =\liminf _{n \rightarrow \infty} \int_{|x| \leqslant R}\left|v_{n}(x)\right|^{\sigma_{c}} d x \geqslant \int_{|x| \leqslant R}\left|v^{*}\right|^{\sigma_{c}} d x,
\end{aligned}
$$

where we have used the weak convergence $v_{n} \rightarrow v^{*}$ in $L^{\sigma_{c}}\left(\mathbb{R}^{N}\right)$ in the last inequality. Using the assumption $\lambda\left(t_{n}\right) / \rho_{n} \rightarrow \infty$ as $n \rightarrow \infty$, we obtain

$$
\liminf _{n \rightarrow \infty} \int_{|x| \leqslant \lambda\left(t_{n}\right)}\left|u\left(x, t_{n}\right)\right|^{\sigma_{c}} d x \geqslant \int_{|x| \leqslant R}\left|v^{*}\right|^{\sigma_{c}} d x,
$$

for all $R>0$, which gives

$$
\liminf _{n \rightarrow \infty} \int_{|x| \leqslant \lambda\left(t_{n}\right)}\left|u\left(x, t_{n}\right)\right|^{\sigma_{c}} d x \geqslant\|V\|_{L^{\sigma_{c}}}^{\sigma_{\sigma_{c}}}
$$

Since $\left\{t_{n}\right\}_{n \in \mathbb{N}}$ is arbitrary, we deduce that

$$
\liminf _{t \rightarrow T^{*}} \int_{|x| \leqslant \lambda(t)}|u(x, t)|^{\sigma_{c}} d x \geqslant\|V\|_{L^{\sigma_{c}}}^{\sigma_{c}}
$$

which completes the proof. 


\section{A remark on another concentration result for the INLS equation}

Here, we present some results which can be obtained in a similar manner to those established in the two previous sections. First, Proposition 4.2 allows us to obtain an alternative GagliardoNiremberg type inequality.

Theorem 6.1. Let $N \geqslant 1,0<b<2, \frac{2-b}{N}<\sigma<\frac{2-b}{N-2}\left(\frac{2-b}{N}<\sigma<\infty\right.$, if $\left.N=1,2\right)$ and $s_{c}=\frac{N}{2}-\frac{2-b}{2 \sigma}$, then the following Gagliardo-Nirenberg inequality holds for all $f \in \dot{H}^{s_{c}} \cap \dot{H}^{1}$

$$
\int_{\mathbb{R}^{N}}|x|^{-b}|f(x)|^{2 \sigma+2} d x \leqslant \frac{\sigma+1}{\|W\|_{\dot{H}^{s_{c}}}^{2 \sigma}}\|\nabla f\|_{L^{2}}^{2}\|f\|_{\dot{H}^{s_{c}}}^{2 \sigma},
$$

where $W$ is a solution to the elliptic equation,

$$
\Delta W+|x|^{-b}|W|^{2 \sigma} W-(-\Delta)^{s_{c}} W=0
$$

with minimal $\dot{H}^{s_{c}}-$ norm.

Sketch of the proof of Theorem 6.1. Step 1. Define the Weinstein functional

$$
J(f)=\frac{\|\nabla f\|_{L^{2}}^{2}\|f\|_{\dot{H}^{s_{c}}}^{2 \sigma}}{\left\||\cdot|^{-\frac{b}{2 \sigma+2}} f\right\|_{L^{2 \sigma+2}}^{2 \sigma+2}}
$$

and the number

$$
J=\inf _{f \in \dot{H}^{s_{c}} \cap \dot{H}^{1}, f \neq 0} J(f) .
$$

Step 2. Let $\left\{f_{n}\right\}_{n \in \mathbb{N}}$ be a minimizing sequence in $\dot{H}^{s_{c}}\left(\mathbb{R}^{N}\right) \cap \dot{H}^{1}\left(\mathbb{R}^{N}\right)$ and consider the sequence $w_{n}(x)=\mu_{n} f_{n}\left(\theta_{n} x\right)$ with

$$
\mu_{n}=\frac{\left\|f_{n}\right\|_{\dot{H}^{s_{c}}}^{\frac{N-2}{2\left(1-s_{c}\right)}}}{\left\|\nabla f_{n}\right\|_{L^{2}}^{\frac{2-b}{2\left(1-s_{c}\right)}}} \quad \text { and } \quad \theta_{n}=\left(\frac{\left\|f_{n}\right\|_{\dot{H}^{s_{c}}}}{\left\|\nabla f_{n}\right\|_{L^{2}}}\right)^{\frac{1}{1-s_{c}}}
$$

Thus, $\left\|w_{n}\right\|_{\dot{H}^{s_{c}}}=\left\|\nabla w_{n}\right\|_{L^{2}}=1$ and $\left\{w_{n}\right\}_{n \in \mathbb{N}}$ is a minimizing sequence in $\dot{H}^{s_{c}}\left(\mathbb{R}^{N}\right) \cap \dot{H}^{1}\left(\mathbb{R}^{N}\right)$.

Step 3. There exists $w^{*} \in \dot{H}^{s_{c}}\left(\mathbb{R}^{N}\right) \cap \dot{H}^{1}\left(\mathbb{R}^{N}\right)$ such that $w_{n} \rightarrow w^{*}$ in $\dot{H}^{s_{c}}\left(\mathbb{R}^{N}\right) \cap \dot{H}^{1}\left(\mathbb{R}^{N}\right)$, as $n \rightarrow \infty$. Moreover,

$$
\left\|w^{*}\right\|_{\dot{H}^{s_{c}}} \leqslant 1 \text { and }\left\|\nabla w^{*}\right\|_{L^{2}} \leqslant 1
$$

and from Proposition 4.2 it follows that $J\left(w^{*}\right)=J$.

Step 4. Since $w^{*}$ is a solution for Euler-Lagrange equation

$$
\left.\frac{d}{d s}\right|_{s=0} J\left(w_{s}\right)=0
$$

where $w_{s}=w^{*}+s \varphi$ for $\varphi \in \mathcal{S}\left(\mathbb{R}^{N}\right)$ we deduce that

$$
\left\langle-\Delta w^{*}-(\sigma+1) J|x|^{-b}\left|w^{*}\right|^{2 \sigma} w^{*}+\sigma(-\Delta)^{s_{c}} w^{*}, \varphi\right\rangle=0,
$$

where $(-\Delta)^{s_{c}} w^{*}$ satisfies $\left\langle(-\Delta)^{s_{c}} w^{*}, \varphi\right\rangle=\left\langle D^{s_{c}} w^{*}, D^{s_{c}} \varphi\right\rangle$ for all $\varphi \in \mathcal{S}\left(\mathbb{R}^{N}\right)$.

Step 5. If $W$ is given by $w^{*}(x)=\alpha W(\beta x)$ with

$$
\alpha=\left[\frac{\sigma^{\frac{2-b}{2\left(1-s_{c}\right)}}}{(\sigma+1) J}\right]^{\frac{1}{2 \sigma}} \quad \text { and } \quad \beta=\sigma^{\frac{1}{2\left(1-s_{c}\right)}},
$$

then

$$
\|W\|_{\dot{H}^{s_{c}}}=\frac{\beta^{\frac{2-b}{2 \sigma}}}{\alpha}\left\|w^{*}\right\|_{\dot{H}^{s_{c}}}=[(\sigma+1) J]^{\frac{1}{2 \sigma}}
$$


and $W$ is a solution (in the weak sense) to the elliptic equation

$$
\Delta W+|x|^{-b}|W|^{2 \sigma} W=(-\Delta)^{s_{c}} W,
$$

with minimal $\dot{H}^{s_{c}}$-norm.

Step 6. Therefore, for all $f \in \dot{H}^{s_{c}}\left(\mathbb{R}^{N}\right) \cap \dot{H}^{1}\left(\mathbb{R}^{N}\right)$

$$
\int|x|^{-b}|f(x)|^{2 \sigma+2} \leqslant \frac{1}{J}\|\nabla f\|_{L^{2}}^{2}\|u\|_{\dot{H}^{s_{c}}}^{2 \sigma}=\frac{\sigma+1}{\|W\|_{\dot{H}^{s_{c}}}}\|\nabla f\|_{L^{2}}^{2}\|f\|_{\dot{H}^{s_{c}}}^{2 \sigma}
$$

which completes the proof.

Next, with this new Gagliardo-Nirenberg type inequality in hand we can prove a variant global well-posedness and concentration results. We will omit the proofs as they are completely analogous to the proofs of Theorem 1.4 and Theorem 1.5.

Theorem 6.2. Let $N \geqslant 3,0<b<\min \left\{\frac{N}{2}, 2\right\}, \frac{2-b}{N}<\sigma<\frac{2-b}{N-2}, s_{c}=\frac{N}{2}-\frac{2-b}{2 \sigma}$ and $\sigma_{c}=\frac{2 N \sigma}{2-b}$. For $u_{0} \in \dot{H}^{s_{c}}\left(\mathbb{R}^{N}\right) \cap \dot{H}^{1}\left(\mathbb{R}^{N}\right)$, let $u(t)$ be the corresponding solution to (1.1) given by Theorem 1.2 and $T^{*}>0$ the maximal time of existence. Suppose that $\sup _{t \in\left[0, T^{*}\right)}\|u(t)\|_{\dot{H}^{s_{c}}}<\|W\|_{\dot{H}^{s_{c}}}$, where $W$ is a solution of the elliptic equation (6.1) with minimal $\dot{H}^{s_{c}}$-norm. Then $u(t)$ exists globally in the time.

From Proposition 4.2 and Theorem 6.1, we also obtain the following $\dot{H}^{s_{c}}$-norm concentration.

Theorem 6.3. Let $N \geqslant 3,0<b<\min \left\{\frac{N}{2}, 2\right\}$ and $\frac{2-b}{N}<\sigma<\frac{2-b}{N-2}$. For $u_{0} \in \dot{H}^{s_{c}} \cap \dot{H}^{1}$, let $u(t)$ be the corresponding solution to (1.1) given by Theorem 1.2 and assume that it blows up in finite time $T^{*}>0$ satisfying (1.6). If $\lambda(t)>0$ is such that

$$
\lambda(t)\|\nabla u(t)\|_{L^{2}}^{\frac{1}{1-s_{c}}} \rightarrow \infty, \text { as } t \rightarrow T^{*},
$$

then,

$$
\liminf _{t \rightarrow T^{*}} \int_{|x| \leqslant \lambda(t)}\left|D^{s_{c}} u(x, t)\right|^{2} d x \geqslant\|W\|_{\dot{H}^{s_{c}}}^{2}
$$

where $W$ is a minimal $\dot{H}^{s_{c}}$-norm solution to elliptic equation (6.1).

Acknowledgments. M.C. was partially supported by Coordenação de Aperfeiçoamento de Pessoal de Nível Superior - CAPES. L.G.F. was partially supported by Coordenação de Aperfeiçoamento de Pessoal de Nível Superior - CAPES, Conselho Nacional de Desenvolvimento Científico e Tecnológico CNPq and Fundação de Amparo a Pesquisa do Estado de Minas Gerais - Fapemig/Brazil.

\section{A Appendix}

Let $N \geqslant 3,0<b<2, \frac{2-b}{N}<\sigma<\frac{2-b}{N-2}$ and $s_{c}=\frac{N}{2}-\frac{2-b}{2 \sigma}$. Given $(q, p) L^{2}$-admissible and $(a, r)$ $\dot{H}^{s_{c}}$-admissible, we define the space (recall definition (2.5))

$$
X=\left(\bigcap_{(q, p) \in \mathcal{A}_{0}} L^{q}\left([-T, T] ; \dot{H}^{s_{c}, p} \cap \dot{H}^{1, p}\right)\right) \bigcap\left(\bigcap_{(a, r) \in \mathcal{A}_{s_{c}}} L^{a}\left([-T, T] ; L^{r}\right)\right)
$$

equipped with the norm

$$
\|u\|_{T}=\|\nabla u\|_{S\left(L^{2} ; I\right)}+\left\|D^{s_{c}} u\right\|_{S\left(L^{2} ; I\right)}+\|u\|_{S\left(\dot{H}^{s_{c} ; I}\right)},
$$

where $I=[-T, T]$. For $m, T>0$, let $S(m, T)$ be the set

$$
S(m, T)=\left\{u \in X ;\|u\|_{T} \leqslant m\right\}
$$


and $d_{T}$ the metric in $X$ given by

$$
d_{T}(u, v)=\|u-v\|_{S\left(\dot{H}^{s} ; I\right)} .
$$

We use a similar argument to the one in Cazenave [5, Theorem 4.4.1] to show the following result.

Lemma A.1. $\left(S(m, T), d_{T}\right)$ is a complete metric space.

Proof. Since $S(m, T) \subset X$ and $X$ is a Banach space, it suffices to show that $S(m, T)$ with the metric $d_{T}$ is closed in $S\left(\dot{H}^{s_{c}} ; I\right)$. For this end, let $\left\{u_{n}\right\}_{n \in \mathbb{N}} \subset S(m, T)$ and $u \in S\left(\dot{H}^{s_{c}} ; I\right)$ such that $d_{T}\left(u_{n}, u\right) \rightarrow 0$, as $n \rightarrow \infty$. This means that $u_{n} \rightarrow u$ in $L_{T}^{a} L_{x}^{r}$ as $n \rightarrow \infty$ for all $(a, r) \dot{H}^{s_{c}}$-admissible and, in particular,

$$
u_{n}(t) \rightarrow u(t) \text { in } L_{x}^{r}\left(\mathbb{R}^{N}\right) \text { as } n \rightarrow \infty \text { for a.a. } t \in[-T, T] .
$$

Moreover, since

$$
\|u\|_{L_{T}^{a} L_{x}^{r}} \leqslant\left\|u_{n}-u\right\|_{L_{T}^{a} L_{x}^{r}}+\left\|u_{n}\right\|_{L_{T}^{a} L_{x}^{r}}, \quad \text { for all }(a, r) \dot{H}^{s_{c}} \text {-admissible, }
$$

and taking $n \rightarrow \infty$, we have

$$
\|u\|_{L_{T}^{a} L_{x}^{r}} \leqslant \liminf _{n \rightarrow \infty}\left\|u_{n}\right\|_{L_{T}^{a} L_{x}^{r}}
$$
we have

Now, let $(q, p) \neq(\infty, 2)$ be a $L^{2}$-admissible pair. Since $\left\{u_{n}\right\}_{n \in \mathbb{N}}$ is a bounded sequence in $L_{T}^{q} \dot{H}^{1, p}$,

$$
u \in L^{q}\left([-T, T] ; \dot{H}^{1, p}\right)
$$

$u_{n} \rightarrow u$ in $L_{T}^{q} \dot{H}^{1, p}$, as $n \rightarrow \infty$ and

$$
\|\nabla u\|_{L_{T}^{q} L_{x}^{p}} \leqslant \liminf _{n \rightarrow \infty}\left\|\nabla u_{n}\right\|_{L_{T}^{q} L_{x}^{p}}
$$

In the same way, we also deduce

$$
\left\|D^{s_{c}} u\right\|_{L_{T}^{q} L_{x}^{p}} \leqslant \liminf _{n \rightarrow \infty}\left\|D^{s_{c}} u_{n}\right\|_{L_{T}^{q} L_{x}^{p}}
$$

We now consider the pair $(q, p)=(\infty, 2)$. From the Sobolev embedding $(2.1)$, we have that $\dot{H}^{s_{c}}\left(\mathbb{R}^{N}\right) \cap \dot{H}^{1}\left(\mathbb{R}^{N}\right) \subset \dot{H}^{s_{c}}\left(\mathbb{R}^{N}\right) \subset L^{\sigma_{c}}\left(\mathbb{R}^{N}\right)$, for $\sigma_{c}=\frac{2 N}{N-2 s_{c}}$. Since the pair $\left(\infty, \frac{2 N}{N-2 s_{c}}\right)$ is $\dot{H}^{s_{c}}$ admissible, we have that (A.1) holds for $r=\sigma_{c}$. In addition, by the definition of $S(m, T)$, we have that $\left\{u_{n}\right\}_{n \in \mathbb{N}}$ is a bounded sequence in $L^{\infty}\left([-T, T] ; \dot{H}^{s_{c}} \cap \dot{H}^{1}\right)$. Thus, by Cazenave [5, Theorem 1.2.5], it follows that

$$
u \in L^{\infty}\left([-T, T] ; \dot{H}^{s_{c}} \cap \dot{H}^{1}\right)
$$

and

$$
\|\nabla u\|_{L_{T}^{\infty} L_{x}^{2}}+\left\|D^{s_{c}} u\right\|_{L_{T}^{\infty} L_{x}^{2}} \leqslant \liminf _{n \rightarrow \infty}\left(\left\|\nabla u_{n}\right\|_{L_{T}^{\infty} L_{x}^{2}}+\left\|D^{s_{c}} u_{n}\right\|_{L_{T}^{\infty} L_{x}^{2}}\right) .
$$

Hence, using (A.2), (A.3), (A.4) and (A.5) for all $(q, p) L^{2}$-admissible and $(a, r) \dot{H}^{s_{c}}$-admissible

$$
\|\nabla u\|_{L_{T}^{q} L_{x}^{p}}+\left\|D^{s_{c}} u\right\|_{L_{T}^{q} L_{x}^{p}}+\|u\|_{L_{T}^{a} L_{x}^{r}} \leqslant \liminf _{n \rightarrow \infty}\left(\left\|\nabla u_{n}\right\|_{L_{T}^{q} L_{x}^{p}}+\left\|D^{s_{c}} u_{n}\right\|_{L_{T}^{q} L_{x}^{p}}+\left\|u_{n}\right\|_{L_{T}^{a} L_{x}^{r}}\right) \leqslant m .
$$

Therefore,

$$
\|\nabla u\|_{S\left(L^{2} ;[-T, T]\right)}+\left\|D^{s_{c}} u\right\|_{S\left(L^{2} ;[-T, T]\right)}+\|u\|_{S\left(\dot{H}^{s_{c} ;[-T, T]}\right)} \leqslant m,
$$

and thus, $u \in S(m, T)$, which completes the proof. 


\section{References}

[1] J. Bergh and J. Löfström. Interpolation spaces. An introduction. Springer-Verlag, Berlin-New York, 1976. Grundlehren der Mathematischen Wissenschaften, No. 223.

[2] J. Bourgain. Global solutions of nonlinear Schrödinger equations, volume 46 of American Mathematical Society Colloquium Publications. American Mathematical Society, Providence, RI, 1999.

[3] L. Campos. Scattering of radial solutions to the inhomogeneous nonlinear Schrödinger equation. arXiv preprint arXiv:1905.02663, 2019.

[4] L. Campos and M. Cardoso. On the critical norm concentration for the inhomogeneous nonlinear Schrödinger equation. arXiv preprint arXiv:1810.09086, 2018.

[5] T. Cazenave. Semilinear Schrödinger equations, volume 10 of Courant Lecture Notes in Mathematics. New York University, Courant Institute of Mathematical Sciences, New York; American Mathematical Society, Providence, RI, 2003.

[6] Y. Cho and M. Lee. On the orbital stability of inhomogeneous nonlinear Schrödinger equations with singular potential. Bull. Korean Math. Soc., 2019.

[7] V. Combet and F. Genoud. Classification of minimal mass blow-up solutions for an $L^{2}$ critical inhomogeneous NLS. J. Evol. Equ., 16(2):483-500, 2016.

[8] F. Demengel and G. Demengel. Functional spaces for the theory of elliptic partial differential equations. Universitext. Springer, London; EDP Sciences, Les Ulis, 2012.

[9] V. Dinh. Scattering theory in a weighted $L^{2}$ space for a class of the defocusing inhomogeneous nonlinear Schrödinger equation. arXiv preprint arXiv:1710.01392, 2017.

[10] V. D. Dinh. Blowup of $H^{1}$ solutions for a class of the focusing inhomogeneous nonlinear Schrödinger equation. Nonlinear Anal., 174:169-188, 2018.

[11] V. D. Dinh. A study on blowup solutions to the focusing $L^{2}$-supercritical nonlinear fractional Schrödinger equation. Journal of Mathematical Physics, 59(7):071506, 2018.

[12] L. G. Farah. Global well-posedness and blow-up on the energy space for the inhomogeneous nonlinear Schrödinger equation. Journal of Evolution Equations, 1(16):193-208, 2016.

[13] L. G. Farah and C. M. Guzmán. Scattering for the radial 3D cubic focusing inhomogeneous nonlinear Schrödinger equation. Journal of Differential Equations, 262(8):4175-4231, 2017.

[14] L. G. Farah and C. M. Guzmán. Scattering for the radial focusing inhomogeneous nls equation in higher dimensions. Bulletin of the Brazilian Mathematical Society, New Series, pages 1-64, 2019. doi: https://doi.org/10.1007/s00574-019-00160-1.

[15] L. G. Farah and B. Pigott. Nonlinear profile decomposition and the concentration phenomenon for supercritical generalized KdV equations. Indiana University Mathematics Journal, 67(5): 1857-1892, 2018.

[16] G. Fibich. The nonlinear Schrödinger equation, volume 192 of Applied Mathematical Sciences. Springer, Cham, 2015. Singular solutions and optical collapse.

[17] F. Genoud. An inhomogeneous, $L^{2}$-critical, nonlinear Schrödinger equation. Journal for Analysis and its Applications, 31:283-290, 2012.

[18] F. Genoud and C. Stuart. Schrödinger equations with a spatially decaying nonlinearity: existence and stability of standing waves. Discrete and Continuous Dynamical Systems, 21(1):137, 2008.

[19] T. S. Gill. Optical guiding of laser beam in nonuniform plasma. Pramana, 55(5-6):835-842, 2000. 
[20] C. D. Guevara. Global behavior of finite energy solutions to the $d$-dimensional focusing nonlinear Schrödinger equation. Appl. Math. Res. Express. AMRX, 2014(2):177-243, 2014.

[21] Q. Guo. A note on concentration for blowup solutions to supercritical Schrödinger equations. Proceedings of the American Mathematical Society, 141(12):4215-4227, 2013.

[22] C. Guzmán. On well posedness for the inhomogeneous nonlinear Schrödinger equation. Nonlinear Anal. Real World Appl., 37:249-286, 2017.

[23] T. Hmidi and S. Keraani. Blowup theory for the critical nonlinear Schrödinger equations revisited. International Mathematics Research Notices, 2005(46):2815-2828, 2005.

[24] J. Holmer and S. Roudenko. On blow-up solutions to the 3D cubic nonlinear Schrödinger equation. Applied Mathematics Research eXpress, 2007(1):Art. ID abm004, 31, 2007.

[25] J. Holmer and S. Roudenko. A sharp condition for scattering of the radial 3D cubic nonlinear Schrödinger equation. Communications in Mathematical Physics, 282(2):42135-467, 2008.

[26] T. Kato. An $L^{q, r}$-theory for nonlinear Schrödinger equations. In Spectral and scattering theory and applications, volume 23 of Adv. Stud. Pure Math., pages 223-238. Math. Soc. Japan, Tokyo, 1994.

[27] F. Linares and G. Ponce. Introduction to nonlinear dispersive equations. Universitext. Springer, New York, second edition, 2015.

[28] C. Liu and V. Tripathi. Laser guiding in an axially nonuniform plasma channel. Physics of plasmas, 1(9):3100-3103, 1994.

[29] F. Merle and P. Raphäel. Blow up of the critical norm for some radial $L^{2}$ super critical nonlinear Schrödinger equations. American Journal of Mathematics, 130:945-978, 2008.

[30] F. Merle and Y. Tsutsumi. $L^{2}$ concentration of blow-up solutions for the nonlinear Schrödinger equation with critical power nonlinearity. J. Differential Equations, 84(2):205-214, 1990.

[31] C. Miao, J. Murphy, and J. Zheng. Scattering for the non-radial inhomogeneous NLS. arXiv preprint arXiv:1912.01318, 2019.

[32] L. Nirenberg. On Elliptic Partial Differential Equations. Annali della Scuola Normale Superiore di Pisa - Classe di Scienze, 13(2):115-162, 1959.

[33] E. Stein and G. Weiss. Fractional integrals on n-dimensional Euclidean space. Journal of Mathematics and Mechanics, pages 503-514, 1958.

[34] C. Sulem and P.-L. Sulem. The nonlinear Schrödinger equation: Self-focusing and wave collapse, volume 139 of Applied Mathematical Sciences. Springer-Verlag, New York, 1999.

[35] T. Tao. Nonlinear dispersive equations, volume 106 of CBMS Regional Conference Series in Mathematics. Published for the Conference Board of the Mathematical Sciences, Washington, DC; by the American Mathematical Society, Providence, RI, 2006. Local and global analysis.

[36] Y. Tsutsumi. Rate of $L^{2}$ concentration of blow-up solutions for the nonlinear Schrödinger equation with critical power. Nonlinear Anal., 15(8):719-724, 1990.

[37] M. Weinstein. Nonlinear Schrödinger equations and sharp interpolation estimates. Comm. Math. Phys., 87(4):567-576, 1982/83.

mykael a. Cardoso, Department of Mathematics, Ufmg, Brazil; Department of Mathematics,

UFPI, BRAZIL

E-mail address: mykael@ufpi.edu.br

LUIZ G. FARAH, Department of Mathematics, UfMG, Brazil

E-mail address: farah@mat.ufmg.br

CARlos M. GUZMán, Department of Mathematics, UfF, Brazil;

E-mail address: carlos.guz.j@gmail.com 